\title{
Ostracoden von Hawaii, insbesondere aus dem marinen Interstitial
}

\author{
G. Hartmann \\ Zoologisches Institut und Zoologisches Museum der Universität Hamburg; \\ Martin-Luther-King-Platz 3, D-W-2000 Hamburg 13, Bundesrepublik Deutschland
}

\begin{abstract}
Ostracods from Hawaii, especially from the marine interstitial system. The present paper deals with ostracods collected by H. Kunz during October and November 1979 on the Hawaiian Islands (Big Island, Maui and Kauai). 26 species were found, nine species are described, two were already known to science, 15 remain in open nomenclature. Although some of the new species are represented only by a single specimen, the morphologic characters permit to distinguish them from other species of the genus. The ostracods of the Hawaiian Islands are very imperfectly known.
\end{abstract}

\section{EINLEITUNG}

Zwischen dem 31. 10. 1979 und dem 17. 11. 1979 wurden von Herrn Helmut Kunz, vormals Universität Saarbrücken, auf den Inseln Kauai, Maui und Hawaii (Big Island) der Hawaii-Gruppe Tiere vor allem des marinen Interstitials gesammelt, unter denen sich auch 26 Ostracoden-Arten befanden. Gewöhnlich wurden diese aus Grundwasserlöchern von Sandstränden geborgen, einige entstammen aber auch aus Gezeiten- oder Spritzwassertümpeln und strandnahen Kleingewässern. Die von Kunz notierten ökologischen Daten sind bei den einzelnen Arten angegeben worden. Obwohl die Ostracoden zum Teil schlecht erhalten (Entkalkung der Schale) und damit der Untersuchung mit Hilfe des REM nicht zugänglich waren, hält der Autor die Publikation der vorliegenden Ergebnisse nicht zuletzt wegen der geringen Anzahl der bislang vorliegenden Daten für wichtig. Erstaunlicherweise sind die Ostracoden der Hawaii-Inseln noch nicht zusammenfassend untersucht worden. Publikationen, die die Hawaii-Inseln direkt betreffen, stammen von Holden (1967, 1976 [Midway-Isld.]) und Kornicker (1976). Holden bearbeitete subfossiles Material von versunkenen Terrassen, Kornicker marine Cypridinacea. Das Litoral ist bislang überhaupt noch nicht nach Ostracoden beprobt worden. Im übrigen liegen nur Funde von Forschungsschiffen von frühen Expeditionen aus dem weiteren Raum der Hawaii-Inseln vor (Brady, vor allem 1880, 1890). Die vorliegenden Aufsammlungen von Kunz können diesen Mangel zwar nicht beheben, sind aber als erster Ansatz einer weiteren Erforschung dieser Inseln zu werten.

Von den insgesamt vorliegenden 26 Ostracoden-Arten waren nur zwei schon bekannt. Von 15 Arten lag nicht genügend Material vor, um an eine Beschreibung zu denken, neun Ostracoden-Arten werden beschrieben und benannt. Im Scan-Bild werden einige Arten ohne nähere Bestimmung abgebildet, um sie für spätere Untersuchungen wiedererkennbar zu machen. 
Verzeichnis der Abkürzungen: $A a=$ Antenna; $A u=$ Antennula; D.ej. $=$ Ductus ejaculatorius; $\mathrm{DR}=$ Dorsalrand der Schale; Endp. $=$ Endopodit $;$ Exp. $=$ Exopodit $_{;}$Fu $=$Furca; HR $=$Hinterrand der Schale; IR = Innenrand der Schalenlamelle; Kfs = Kaufortsätze; Kop.-Org. = Kopulationsorgan

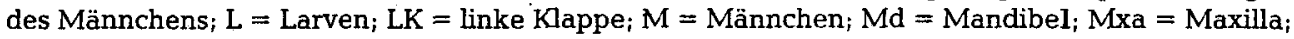
$\mathrm{Mxu}=$ Maxillula Ovd. $=$ Ovidukt $;$ P 1, 2, $3=$ Schreitbeine $(\mathrm{P} 1=\mathrm{Mxa}) ; \mathrm{RK}=$ rechte Klappe; $\mathrm{S}=$ Saum; $\mathrm{Sb}=$ Schwimmborsten; $\mathrm{SIm}=$ Schließmuskelfeld; UR = Unterrand der Schale; VL = Verwachsungslinie $; \mathrm{VR}=$ Vorderrand der Schale; VZ = Verschmelzungszone; W = Weibchen; I, II, III etc. (römische Ziffern allein) = 1., 2., 3. etc. Glied einer Gliedmaße.; ZIM = Zoologisches Institut und Zoologisches Museum Hamburg.

SYSTEMATISCHER TEIL

Unterklasse Myodocopa Sars, 1866

Ordnung Cladocopa Sars, 1866

Familie Polycopidae Sars, 1866

Gattung Polycope Sars, 1866

Polycope hawaiiensis sp. $\mathrm{n}$.

(Abb. 1-10)

N a me: Nach dem Fundort der Art, Hawaii.

Holotypus: ZIM K - 34636

Paratypen: ZIM K $-34637-638$

Locus ty picus: Kawai-hae Bay, Spencer Beach.

Material: 14 Exemplare von Hawaii, 17 Exemplare von Kauai.

Beschreibung: Die Schalen waren schlecht erhalten und können bei der Präparation leicht deformiert worden sein. Sie sind, wie bei allen Polycopiden, nahezu kreisrund, im Schloßbereich abgeflacht und am VR leicht eingebuchtet. Am VR der RK, unter der Einbuchtung, befindet sich eine Reihe feiner Zähnchen, die bis in die Mitte des UR reichen. An der LK sind diese in bestimmten Abständen mit verstärkten Zähnchen durchsetzt. Der Saum hat die Breite der Zähnchenreihe und reicht, sich in Seitenansicht verschmälernd, bis in den HR hinein. Randkanäle waren nur wenige an der RK zu sehen (vgl. Abb. 1, 2). Das Bellonci-Organ konnte wegen der geringen Größe der Tiere nicht gefunden werden. Es bleibt daher die Zuordnung zu einer anderen Gattung der Familie, evtl. Micropolycope, zweifelhaft. Au mit deutlichem dorsalen Vorsprung, auf dem eine kurze Borste sitzt. Endteil der Basis ventral leicht geschwungen. Endglied klein. Grundglied der Aa fast dreieckig, ventral, vor dem Endopoditen, verstärkt. Md mit zweizähniger Kaulade. Exopodit birnförmig, mit behaarter Spitze. Neben dem Exopoditen steht eine Borste, die diesen an Länge übertrifft (s. Abb. 5-7). Mxu mit hervorspringenden proximalen Enditen, der 5 Borsten trägt. Endborsten der Gliedmaßen lang. Exopodit der 5. Gliedmaße ist eine an der Basis verdickte Borste. Fu beiderseits mit sieben Borsten, dahinter ein nach vorn gerichteter Dorn. Im Eileiter waren 1-2 fertige Eier, was auf die Geschlechtsreife der sehr kleinen Tiere hinweist.

$M a B e$ : Länge 0,14-0,15 mm; Höhe 0,13-0,14 mm.

Fundorte: Hawaii - Anaeho'omalu Bay, Hauptstrand, unter NWL;

- Kawai-hae Bay, Spencer Beach, ruhiges Wasser, Mittelsand mit Feinmaterial, $1 \mathrm{~m}$ unter NWL, Sandoberfläche; 
- ebendort, Küstengrundwasserloch $15 \mathrm{~cm}$ tief, $0,5 \mathrm{~m}$ vom Spülsaum (Locus typicus).

Kauai - Anini Community, Beach Park, Grobsand aus dem Meer, $7 \mathrm{~m}$ von Ufer, $40-50 \mathrm{~cm}$ Wassertiefe.

Ökologie: Die Art bewohnt marine Lückensysteme verschiedener Korngröße und Tiefe im Sediment.

B e zi e h u n g e n: Da das Bellonci-Organ nicht gefunden wurde, ist die Zuordnung zu der Gattung nicht gesichert. Möglicherweise handelt es sich um eine Art der Gattung Micropolycope. Die Art ist die bislang kleinste der Familie Polycopidae.

Polycope (?Polycopiella) spec. Maui 11

(Abb. 11-15)

Von dieser Art wurde nur ein geschlechtsreifes Weibchen in einer Probe von Maui gefunden. Das Weibchen hatte ablagereife Eier im Ovidukt.

Material: ZIM K- 34639

Beschreibung: Schale fast kreisrund, im Schloßbereich abgeflacht und am oberen VR leicht eingebuchtet. Über der VR-Einbuchtung entspringt nahe dem Schalenrand eine Borste. Der untere VR und UR erscheinen fein gestreift - feine Zähnchen vor einem häutigen Saum. Au gestreckt, Grundglied kaum abgesetzt, Dorsalrand mit schwacher Borste. Endp. der Aa kurz, Endglied mit Basisglied verschmolzen. An der Md wurde als Exp. nur eine Borste nachgewiesen, die an einem behaarten Randteil entspringt. Mxu und Mxa ohne besondere Kennzeichen. Furca symmetrisch mit je 7 Klauen.

Länge des Weibchen $0,21 \mathrm{~mm}$.

Fundorte: Maui - Honokowai (nördlich Ka'anapali), Küstengrundwasserloch, $2 \mathrm{~m}$ vom Spülsaum, $40 \mathrm{~cm}$ Tiefe, Grobsand.

B e zi e h u n gen : aufgrund der unvollständigen Beschreibung nicht diskutiert.

Familie Bairdiidae Sars, 1866

In verschiedenen Proben von Hawaii, Maui und Kauai fand sich Schalenmaterial, dessen weitere Bearbeitung nicht möglich ist.

\section{Superfamilie Cytheroidea Baird, 1850}

In die "Sammelfamilie" Cytheridae Baird, 1866 gehört vermutlich eine Art, die mit Larven und Subadulti in einer Probe von Molukai (Kaunakakai) gefunden wurde. Auf einem wattähnlichen Strand, der, durch ein Riff geschützt, Stillwasser aufwies, war eine Population dieser nicht bestimmbaren Art in größerer Individuenzahl vorhanden.

Kurzbeschreibung: Au 6gliedrig, Aa mit zwei Endklauen, Md-Taster wie Cytheridae, Atemplatte mit 4 Strahlen unterschiedlicher Länge. Mxu und Schreitbeine ohne besondere Kennzeichen, einfach. Schale entkalkt, Rand vorn mit spärlichen, hinten mit dicht stehenden Randkanälen.

F u n d o r t e : Kaunakakai, Feinsand mit Schluff, $20 \mathrm{~cm}$ unter Meeresspiegel. 
Familie Trachyleberidiae Sylvester-Bradley, 1948

Aus verschiedenen Proben von Maui lag unbestimmbares Schalenmaterial vor.

Familie Hemicytheridae Puri, 1953

Hemicytheride spec. Ka 29

(Tafel 1: Fig. 1-2)

Von Kauai, Kekaha, Bachmündung, liegen zwei Schalen einer unbekannten Hemicytheride (?) vor. Sie werden hier abgebildet. Es handelt sich um stark verkalkte, vor allem in der terminalen Hälfte mit Rippen versehene Schalen mit einfachen Porenkanälen. Die Schalen haben ein amphidontes Schloß.

Länge der Schalen 0,51 mm.

Familie Cobanocytheridae Schornikov, 1975

Gattung Paracobanocythere Gottwald, 1983

Paracobanocythere hawaüensis Gottwald, 1983

Erwähnt wurde die Art erstmals von Gottwald in seiner Dissertation aus dem Jahre 1980 als "sp. nov.". Da Gottwald die Art später (1983) an anderer Stelle nochmals in einer regulären Zeitschrift als "sp. n." beschreibt, ist die erste Nennung hinfällig. Da die Dissertation z. T. andere Namen als die definitive Publikation enthält und dadurch nomenklatorische Verwirrung angerichtet wird, sollte die Nomenklaturkommission endlich eine klare Entscheidung über den Wert von Dissertationen hinsichtlich nomenklatorischer Bindung finden.

Gottwalds Art kam auf Hawaii, Kauai und Mani in verschiedenen Proben vor. Die Exemplare zeigten volle Übereinstimmung mit Gottwalds Originalbeschreibung.

Fundorte : Hawaii - Kiholo-Bay, Schill vom Strand, Lavasteine, hoher Strandwall: 1 Exemplar;

- Kawaihae-Bay, Spencer Beach, ruhiges Wasser, Mittelsand mit Feinmaterial, Spülsaum mit etwas groberem Material: ZIM K-34640, Population;

- Küstengrundwasser, $15 \mathrm{~cm}$ tief, 0,5 m vom Spülsaum: $6 \mathrm{~W}$.

Kauai - Kehaha, Bachmündung ins Meer, schwarzer Lavasand mit Olivin; mittlerer Grobsand, Prallhang (Seeseite): $1 \mathrm{M}, 4 \mathrm{~W}$, $1 \mathrm{~L}$; grober Grobsand, NW-Linie: $4 \mathrm{M}, 8 \mathrm{~W}_{i}$ Sand zwischen Lavaklippen: $1 \mathrm{M}$;

- Anini, Community Beach Park, Grobsand aus dem Meer, $7 \mathrm{~m}$ vom Ufer, $40-50 \mathrm{~cm}$ Wasserbedeckung: $1 \mathrm{M}$, 1 Subad.; Material: ZIM K-34641-642

Maui - Strand südlich Lahaina, SW-Seite von Westmaudi; grober Grobsand aus dem Meer, $30 \mathrm{~cm}$ unter MWL vom Hang: $6 \mathrm{~W}$;

- Ka'anapali, nördl. Lahaina, mittlerer Grobsand beim Hotel Maui Kai, Mittelwasser bei auflaufender Flut: $3 \mathrm{~W}$. 
Ökologie: Die Art bevorzugt offenbar grobe Substrate und kommt sowohl im Meer als auch im Küstengrundwasser im Lückensystem vor.

\section{Gattung Cobanocythere Hartmann, 1959 \\ Cobanocythere konensis sp. $\mathrm{n}$.}

(Abb. 16-24)

Na me : Nach dem Fundort der Art, dem Distrikt Kona auf Hawaii, Big Island.

Holotypus: ZIM K-34643

Paratypen: ZIM K-34643

Locus typicus: City of Refuge, südlich von Kailua, Kona. Sandstrand nahe Tempel, Schill.

Material: $2 \mathrm{~W}, 1 \mathrm{M}$.

Beschreibung: Schalen vom Männchen und Weibchen übereinstimmend, im Umriß schlank, nach hinten-ventral zugespitzt. Größte Höhe deutlich vor der Mitte. Die weiteste Vorwölbung liegt vorn unter der halben Höhe. Am vorderen UR bewirkt eine Einbuchtung ein lippenartiges Vorspringen des Vorderendes (labiata-Gruppe). Dort tritt der Schalenrand deutlich zurück (Einzelheiten s. Abb. 17). In der Ansicht von oben ist die Schale tropfenförmig gestaltet, das Vorderende zugespitzt, das Hinterende gerundet. Die größte Breite liegt etwa in der Mitte. Am Schalenrand sind Randkanäle nur am Vorderende sichtbar. Es sind wenige, große, einfache Flächenkanäle vorhanden.

Au kräftig, 6gliedrig. Das Grundglied ist gegen den Endteil stark angewinkelt. Beborstung: III distal-ventral eine, IV etwas distal der Mitte ventral (und dorsal?) je eine Borste, distal-ventral eine, dorsal 3. Am vorletzten und am kleinen Endglied stehen zusammen 4 Borsten. Alle Borsten sind kürzer als die letzten 3 Glieder. Auch die Aa ist kräftig. II trägt eine starke Spinnborste, die das Ende der Gliedmaße überragt. III nur ventral-distal mit kurzer Borste, IV mit einer starken und einer schwächeren Borste, die (?) scherenartig gegeneinander stehen. Md mit breiter Kaulade, deren innerer Zahn am kräftigsten ist. Zwischen diesem und dem viel kleineren 2. Zahn steht eine Borste. Die anderen 5 Zähne sind klein. Taster schlank, die Beborstung war nicht klar erkennbar (s. Abb. 21). Mxu mit schlanken Kfs und Taster, die lange, feine Borsten tragen. Schreitbeine relativ kurz, Endteil 3gliedrig. Das Stammglied trägt Knieborsten. Die übrige Beborstung war nicht erkennbar. Klauen kurz und spitz. Sehr charakteristisch ist das männliche Kopulationsorgan gebaut. Rechte und linke Seite sind verschieden, eine Hälfte trägt einen abgerundeten, die andere einen zugespitzten Aufsatz.

M a Be : Länge 0,26-0,27 mm, Höhe $0,08 \mathrm{~mm}$, Breite des Gehäuses 0,15 mm.

Fund orte: s. Locus typicus.

Ökologie: s. Locus typicus.

Beziehungen: Die Art gehört zur labiata-Gruppe der Gattung und ist durch ihre Schalenform, Größe und besonders den Bau des männlichen Kopulationsorgans von anderen Arten leicht zu trennen. Es wird auf die zusammenfassende Betrachtung von Gottwald $(1980,1983)$ verwiesen. 


\section{Cobanocythere spec. HW 18}

(Abb. 25)

In der Kawaihae Bay, Spencer Beach, wurde ein Weibchen einer noch unbekannten Art gefunden, das wegen des schlechten Erhaltungszustands nicht beschrieben werden kann. Die Schale wird aber abgebildet. Das Tier hatte eine Länge von 0,24 mm und eine Höhe von $0,105 \mathrm{~mm}$. Die Art kam in ruhigem Wasser auf Mittelsand, der mit Feinmaterial durchsetzt war, in $1 \mathrm{~m}$ Tiefe vor.

Familie Psammocytheridae Klie, 1938

Gattung Psammocythere Klie, 1938

Psammocythere hawaiiensis sp. $\mathrm{n}$.

(Abb. 26-33)

$\mathrm{N}$ a m e : Nach dem Fundort der Art, Hawaii (Big Island).

Holotypus: ZIM K-34644, Männchen.

Paratypen: ZIM K-34645-647, 2 M, 6 W.

Locus typicus: Anaeho'omalu, Hauptstrand, Probe aus dem Meer unterhalb MWL.

Material: s. Holo- und Paratypen.

B e s chreibung: Schalen von $M$ und $W$ nicht unterschieden, langgestreckt, mit fast parallelen DR und UR. Ventral wird der UR, besonders in der vorderen Hälfte der Schale, von der Schalenoberfläche in Seitenansicht überwölbt. Die VZ ist besonders im dorsalen Teil von VR und HR breit ausgebildet, mit unregelmäßig angeordneten Porenkanälen unterschiedlicher Länge. Flächenkanäle liegen spärlich und unregelmäßig über die gesamte Schalenoberfläche verteilt; z. T. tragen sie kurze Borsten.

Au mit 3gliedrigem Endteil. Lange, geringelte $\mathrm{Sb}$ tragen nur das kurze quadratische Endglied und das zylindrische vorletzte Glied. An diesem steht median-ventral zusätzlich eine kurze Borste (vergl. Abb. 29). Aa wie bei $P$. remanei, Einzelheiten schwer erkennbar. Md mit kräftiger, mit starken Zähnen und Stacheln besetzter Kaulade und kräftigem Taster (Abb. 27, 28). Das Grundglied trägt ventral eine einseitig bestachelte und 2 glatte Borsten, II ventral distal 2 lange, glatte Borsten, dorsal, proximal eine feine, schwer sichtbare. III ist ventral-distal, nach der Erweiterung des Gliedes, mit 2, dorsal, auf besonderem Absatz, ebenfalls mit 2 Borsten bewehrt. Das zylindrische Endglied besitzt distal 3 Borsten. Mxu mit kräftigen Kfs und Taster. Letzterer besitzt ein schmales Endglied mit 2 kräftigen Klauen am Ende. Die Klauen der Kfs sind kräftig, ihre genaue Anzahl war nicht zu ermitteln. Ventral entspringen an der Basis von Kfs II 2 Borsten, eine steht an der Basis des Tasters. Mxa ist ein Greiforgan, dessen distale Borste gegen eine mit einer Borste versehene Erweiterung des II schlägt. Schreitbeine schlank, mit 4gliedrigem Distalteil. Klaue relativ kurz und kräftig. Besonders charakteristisch ist wieder das Kopulationsorgan des Männchens gebaut. Es besitzt einen löffel- und einen zipfelförmigen Aufsatz und einen hakenartigen, seitlichen Fortsatz. Das zentrale Chitinspangenwerk ist kaum darstellbar. In der Skizze (Abb. 33) wurden nur die prominentesten Spangen abgebildet. 
Maße: Männchen und Weibchen Länge $0,37 \mathrm{~mm}$, Höhe $0,11 \mathrm{~mm}$.

Fund or te : Locus typicus und ein W ebendort aus Mittelsanden eines Strandtümpels; ferner: Kawaihae Bay, Spencer Beach, Brandungszone, grober Mittelsand mit Feinmaterial aus Spülsaum: ein $\mathrm{M}$.

Ökologie: Die Art bewohnt offenbar insbesondere die Oberfläche von Mittelsanden.

Beziehungen: Es sind insgesamt bisher drei Arten bekannt, davon eine fossil aus China ( $P$. luminosa). Die Typus-Art stammt von Helgoland, eine weitere von den Galápagos-Inseln ( $P$. santacruzensis Gottwald, $1983=P$. hartmanni Gottwald, 1980). Alle drei unterscheiden sich sowohl im Schalenbau als auch im Bau der Extremitäten und - soweit bekannt - im Bau des männlichen Kopulationsorgans. Die Gattung dürfte in geeigneten Lebensräumen weltweit verbreitet sein.

\title{
Familie Loxoconchidae Sars, 1925 \\ Gattung Loxoconcha Sars, 1966 \\ (Tafel 1: Fig. 3-10, Tafel 2: Fig. 11-14a)
}

\begin{abstract}
Aus einer Probe von der Bachmündung bei Kekaha (Kauai) lagen die Schalen von 3 Loxoconcha-Arten vor, die keiner bekannten Spezies zugeordnet werden können. Ebenfalls von dort stammt ein entkalktes Exemplar mit Weichkörper. Offensichtlich gehören diese nicht zu der Interstitialfauna des Strandes, sondern sind eingeschwemmt. Darauf weist auch die Fundstelle, inneres Bachufer, hin.

Obwohl die Arten nicht zugeordnet oder beschrieben werden können, sollen die Scanphotos derselben, die ein Wiedererkennen sicherlich ermöglichen, abgebildet werden: Loxoconcha spec. Ka 1, 2 Klappen von Adulti, 6 Klappen von Larven; (Tafel 1: Fig. 5-10, Tafel 2: Fig. 11-12). Loxoconcha spec. Ka 2, 2 LK von Adulti (Tafel 2: Fig. 13-14a); Loxoconcha spec. Ka 3, 2 Klappen eines Adulten (Tafel 1: Fig. 3-4). Von Maui, Honokowai, lagen weitere Exemplare der Gattung Loxoconcha aus einem Fluttümpel vor, die aber wegen der schlechten Erhaltung ebenfalls nicht beschrieben werden können.
\end{abstract}

\section{Familie Pectocytheridae Hanai, 1957 \\ Gattung Morkhovenia Teeter, 1975 \\ Morkhovenia inconspicua (Brady, 1880)}

(Tafel 2: Fig. 14b)

Die Art ist offenbar zirkumtropisch auf Korallenriffen verbreitet. Das mir vorliegende Material stammt von Kauai, einmal aus der schon erwähnten Bachmündung bei Kekaha (s. Loxoconcha-Arten), zum anderen aus Grobsand aus $40-50 \mathrm{~cm}$ Tiefe, $7 \mathrm{~m}$ vor dem Community Beach Park von Anini.

Die Art wird noch einmal abgebildet. 
Familie Cytheruridae G. W. Müller, 1894

Unterfamilie Cytheropterinae Hanai, 1957

Gattung Cytheropteron Sars, 1866

?Cytheropteron spec. Maui 16

(Abb. 34-44)

Ein leider sehr schlecht erhaltenes Weibchen, das ich nur mit Bedenken der Gattung Cytheropteron zuordne, fand sich in einer Probe von Kalepolepo (bei Kihei, Westküste von Maui). Sie wurde aus einem Ebbetümpel zwischen Riff und Land aus MittelFeinsand genommen. Die erhaltenen Teile wurden gezeichnet.

Beschreibung: Eine Art mit fast parallelen DR und UR, mit deutlichem Kaudalfortsatz, der über halber Schalenhöhe liegt. Bemerkenswert ist eine sehr breite VZ am VR, die von unregelmäßig angeordneten sehr feinen Randkanälen durchzogen wird. Flächenkanäle einfach, mit kurzen Borsten. Slm mit 4 Narben in senkrechter Reihe und 2 weiteren davor. Au schlank, vor allem terminal beborstet (Abb. 36). Aa mit 3gliedriger, sehr kräftiger Spinnborste, auffällig ventral eingebuchtetem II, 2 Terminalklauen und langem vorletzten Glied. Md-Taster kurz, gedrungen, mit kleinem Endglied und einem Strahl an der Atemplatte. Kaulade schmal. Mxu mit 2 mundwärts gerichteten Strahlen an der Atemplatte, Taster mit kurzem Endglied, länger als die Kfs. Schreitbeine kräftig, mit terminal stark gekrümmten Klauen. Die Beborstungsformel des Grundglieds ist: $121 /$ $210 / 010$. Körperende des Weibchens lang ausgezogen, mit flachem Genitalfeld. Im Ovd. befanden sich ablagereife Eier.

$\mathrm{Ma} \mathrm{Be}$ : Länge 0,35 mm, Höhe 0,15 mm.

Bezi ehungen: Die Randverhältnisse (VZ) weichen von Cytheropteron ab. Die Art ist für die Gattung sehr klein. Sie gehört abeŕ dennoch vermutlich nicht der Interstitialfauna an.

Kein Museumsmaterial.

Familie Paracytherideidae Puri, 1957

Gattung Paracytheridea G. W. Müller, 1894

Paracytheridea spec. Kaui 29

(Tafel 2: Fig. 15)

Aus der Probe bei der Bachmündung bei Kekaha (s. Loxoconcha-Arten) lag auch die Schale einer Paracytheridae-Art vor, die kommentarlos abgebildet wird.

Familie Xestoleberididae Sars, 1928

Gattung Xestoleberis Sars, 1866

Xestoleberis hawaiiensis sp. $\mathbf{n}$.

(Abb. 45-56)

Na me: Nach dem Fundort der Art, der Insel Hawaii (Big Island).

Holotypus : ZIM K-34648, ein Männchen. 
Par atypen: ZIM K-34648, 2 Schalen.

Locus typicus: Grober Schill und Korallenschutt aus einem vom Meer abgeschnittenen Fluttümpel mit schwachem .Algenbewuchs und Fischen; Kealia Beach = Paianihi Beach.

Material: Holotypus und Paratypen

Beschreibung des Holotypus: VR und HR gleichmäßig gerundet mit in Seitenansicht weitester Vorwölbung unter der halben Höhe. Der DR ist flach gekrümmt und hat seine größte Höhe hinter der halben Länge. Der UR ist in der Mundgegend eingebuchtet. Die VZ ist am VR etwas breiter als am HR und wird von vielen RK durchzogen (s. Abb. 45). Der IR war nur am VR, nicht weit von der VZ entfernt, auszumachen. Der nierenförmige Fleck ist klein, senkrechte Pfeilerchen scheinen zu fehlen (Entkalkung?). Die Randverhältnisse waren nicht genau erkennbar.

Die Au besitzt 6 Glieder, der Endteil ist kräftig gebaut. Beborstung: II ventral-distal eine, III dorsal-distal eine, IV dorsal-distal 2, V dorsal-distal 2, ventral-distal eine, Endglied mit 3 Borsten und einem Sinnesstab. Die Borsten nehmen terminal an Länge zu. Aa sehr kräftig, mit starker Spinnborste, distal-ventral steht am II nur eine kurze Borste, median am III finden sich dorsal eine, ventral 2 Borsten, III ist distal mit einer kräftigen Klaue bewehrt, das Endglied mit 2 derselben, die als Subchela arbeiten (?): Eine der Klauen ist dorsal gerieft. Md mit starkem Taster und schmaler, mit großen Zähnen besetzter Kaulade. Die Atemplatte trägt einen Strahl. Die beiden Grundglieder des Tasters sind gestreckt, das Endglied ist quadratisch. Das Grundglied besitzt distal-dorsal eine, ventral 2 schwache Borsten. Am II steht dorsal-median eine Borste, ventral-distal eine Klaue. Das Endglied besitzt eine kräftige Klaue und vermutlich 3 weitere Borsten. Mxu mit einem behaarten, kurzen aberranten Strahl an der Atemplatte. Taster und Kfs kräftig. Tasterendglied fast so lang wie die Basis, distal mit einer starken Klaue und einer Borste bewehrt. Borsten der Kfs besonders am Kfs I kurz und klauenförmig, am II und III borstenähnlicher. Schreitbeine kurz, gedrungen, mit kräftigen, hakenartigen Klauen bewehrt. Distale Borste von II nur am P 3 so lang wie, sonst kürzer als das folgende Glied. Die Beborstung der Grundglieder folgt der Formel: 1 1(?) $1 / 111 / 111$. Körperende des Weibchens spitz; Fu mit zwei ungleich langen Borsten, Geschlechtsfeld ragt nicht vor. Das Kopulationsorgan des Männchens besitzt - wie bei vielen Arten dieser Gattung einen dreieckigen Aufsatz. Die Kapsel ist in einen schmaleren distalen und einen breiteren Basalteil geteilt. Ein starkes Spangenwerk ist an der Basis des Aufsatzes entwickelt (s. Abb. 56).

$\mathrm{Ma}$ a e : Länge $0,42 \mathrm{~mm}$, Höhe $0,21 \mathrm{~mm}$.

Fundorte: s. Locus typicus.

Be zi e h u n ge n: Die Art gehört zu den kleineren Arten der Gattung. Charakteristisch ist der Schalenumriß, der nierenförmige Fleck, die Randzone der Schale, der Bau von $\mathrm{Au}, \mathrm{Md}$ und $\mathrm{Mxu}$ sowie das männliche Kopulationsorgan. Im Bereich des IndoWestpazifiks war eine ähnliche Art nicht nachweisbar.

Xestoleberis spec. Ma 20

Aus einer Probe von Ke'anae (NO-Küste von Maui) lag die Schale einer Xestoleberis-Art vor, die nicht zugeordnet werden kann. 
Familie Paradoxostomatidae Brady \& Norman, 1889

\author{
Gattung Paradoxostoma Fischer, 1855 \\ Paradoxostoma kunzi sp. $\mathrm{n}$.
}

(Abb. 57-65b)

Na me : Der Autor widmet die Art Herrn Helmut Kunz, vormals Saarbrücken, jetzt Bischmisheim. Herr Kunz hat das vorliegende Material gesammelt.

Holotypus: ZIM K-34649, ein Männchen.

Locus typicus: Lookout zwischen Kapa'a und Ke'alia (Ostküste von Kauai), grober Schill mit Korallenstücken.

Material: kein weiteres Material.

Beschreibung: Schale langgestreckt, hinten deutlich höher als vorn. VR schmal gerundet, HR etwas breiter mit kurzem vertikalen Abschnitt am Schalenende. VZ vorn schmal, hinten breiter, mit nur wenigen Randkanälen. IR, wegen der Entkalkung der Schale, nicht sichtbar. Die 4 Endglieder der Au verhalten sich in ihren Längen (am DR gemessen) wie $6: 4: 1,7: 0,8$. Die schwache Beborstung war schlecht sichtbar (s. Abb. 59). Die Aa besitzt ein sehr kräftiges Basalglied. Auch II ist ungewöhnlich stark, distal etwas schmaler als proximal. III und VI verhalten sich in ihren Längen (am Ventralrand gemessen) wie $6: 4$. Das Endglied ist klein. II, III und das Endglied tragen ventral je eine Borste, die kürzer als das folgende Glied ist. Am Endglied findet sich außerdem eine kräftige Klaue. Die Spinnborste ist so lang wie der Endteil der Aa. Die Spinndrüse ist deutlich zweilappig. An der Mxu entspringen an der Atemplatte 2 gleichlange mundwärts gerichtete Strahlen. In der Saugscheibe des Mundes waren nur die Spitzen der Stechborste der Md sichtbar (Abb. 58). Die Schreitbeine besitzen ein grobes Basalglied, dem ein relativ schwacher Gehteil ansitzt. Die kurzen, nur am Ende etwas aufgerichteten Klauen sind innen fein gestachelt. Die Beborstung der Grundglieder folgt der Formel $110 / 010 / 010$. Die Knieborste von P 1 ist zwar kräftiger als die der anderen Schreitbeine, für Männchen dieser Gattung aber auffällig schwach. Die distal-dorsalen Borsten von II sind kürzer als III, am längsten am P 1, am kürzesten am P 2. Die Beschreibung der Art nach nur einem männlichen Exemplar erfolgte wegen der sehr charakteristischen Ausbildung des Kopulationsorgans. Dieses besitzt einen großen messerschneidenförmigen Aufsatz, vor dem 2 weitere Fortsätze stehen, ein dornförmiger und ein löffelförmiger (Abb. 65a, b).

$\mathrm{MaBe}$ : Länge $0,35 \mathrm{~mm}$, Höhe $0,105 \mathrm{~mm}$.

Fund orte: s. Locus typicus.

Beziehungen: Die Art gehört zu den kleinsten der Gattung. Die Schalenform und insbesondere die einmalige Ausbildung des Kopulationsorgans machen ihre Unterscheidung einfach. Von den Inseln des Pazifiks sind bislang nur 6 Arten der Gattung bekannt (vgl. Hartmann, 1984). Die Zahl der Arten von Paradoxostoma dürfte weit höher liegen.

Paradoxostoma kauaiensis sp. $\mathrm{n}$.

(Abb. 66-81)

$\mathrm{N}$ a m e: Nach dem Locus typicus der Art, der Insel Kauai.

Hol otypus: ZIM K-34650, ein Weibchen.

Allotypus : ZIM K-34651, ein Männchen. 
Locus typicus: Lookout zwischen Kapa'a und Kea'lia (Ostküste von Kauai), grober Schill mit Korallenstücken.

Locus all otypicus: Napili-Bay (NW-Seite von Maui), Fluttümpel mit Fischen.

Material: neben den Typen noch eine Larve von Kekaha (Sand zwischen Lavaklippen) auf Kauai.

B es chreibung: Schalen von Männchen und Weibchen im Umriß übereinstimmend, das Weibchen ist etwas kleiner. Die Art gehört zu den hohen Formen, deren größte Höhe am flach gewölbten DR etwa in der Mitte liegt. Die weiteste Vorwölbung des HR liegt an der Grenze von DR und HR. Von dort fällt der HR steil nach vorn geneigt zum fast geraden UR ab. Das Vorderende ist schmal gerundet, seine weiteste Vorwölbung liegt unter halber Höhe. Ein Saum ist im gesamten Bereich des VR, UR und HR in Seitenansicht sichtbar. Das Slm besteht aus vier großen Narben. Beim Männchen ist eine große Drüse in der hinteren Schalenhälfte sichtbar. Die VZ ist vorn etwas schmaler als hinten; dies ist deutlicher beim Weibchen. Sie wird in unregelmäßigen Abständen von RK durchzogen. Flächenkanäle sind spärlich vorhanden (Abb. 66, 72). Die Au ist beim Männchen und Weibchen leicht abweichend gebaut. Ihre Endglieder verhalten sich in ihren Längen (am Dorsalrand gemessen) beim Weibchen wie: $55: 65: 30: 20$, beim Männchen wie $55: 58: 40: 15$. Beim Männchen standen ventral am II median eine, am III distal eine, am IV distal 2, am V distal eine und terminal 3 Borsten. Dorsal distal waren nur IV und V mit je einer Borste versehen. Beim Weibchen (vgl. Abb. 68) waren die Verhältnisse ähnlich, es konnten aber nicht alle Borsten nachgewiesen werden. Die Aa beider Geschlechter stimmten völlig überein. Bemerkenswert sind eine sehr dicke 3gliedrige Spinnborste und eine zweispitzige Terminalklaue (Abb. 69). Die Spinndrüse ist einfach (Abb. 74). Md und Mxu bei beiden Geschlechtern weitgehend übereinstimmend. Der Taster ist zart, distal sind 2 kleine Glieder abgetrennt. Er ist mit 5 langen Borsten versehen. Die zur Stechborste umgewandelte Kaulade ist kräftig und steckt proximal in einem umfangreichen Muskelpaket. An der Mxu ist der innere Kfs beim Männchen mit 2 (Abb. 76), beim Weibchen nur mit einer Borste (Abb. 70) bewehrt. Auch die Schreitbeine stimmen in beiden Geschlechtern weitgehend überein. Die kräftigen Basalglieder sind nach der Formel $110 / 110 / 010$ bewehrt. Die Knieborsten beider Geschlechter sind am P 1 verstärkt (Abb. 71, 77). Die Schreitteile sind schlank, die Klauen innen fein gestachelt. Die distal-dorsalen Borsten von II sind kürzer als das folgende Glied, am P 3 winzig. Das bürstenförmige Organ besitzt einen Haupt- und einen schwachen Nebenast. Sehr charakteristisch ist wieder das männliche Kopulationsorgan gebaut (Abb. 81), dessen äußerer Aufsatz in eine Borste mündet. Der innere Aufsatz ist löffelartig. Beide liegen einander eng an.

Maße: Männchen Länge $0,42 \mathrm{~mm}$, Höhe $0,21 \mathrm{~mm}$. Weibchen Länge $0,385 \mathrm{~mm}$, Höhe $0,21 \mathrm{~mm}$.

Fundorte: s. Locus typicus und Locus allotypicus.

Beziehungen: Die Art ist durch den Bau der Aa und des Kopulationsorgans leicht von anderen zu unterscheiden. Auch sie gehört zu den kleinen Arten der Gattung. Auf P. kunzi sp. n. (s. o.) wird verwiesen.

Paradoxostoma spec. Maui 5

Ein weibliches Exemplar fand sich in einem Fluttümpel in der Napili-Bay von Maui (NW-Seite der Insel, Grund mit Kies und Steinen). 


\section{Paradoxostoma spec. Maui 10}

In Rotalgen, die auf Lavablöcken bei Honokowai (Maui) wuchsen, kam eine weitere Art vor, von der aber nur larvale Exemplare und Schalen gesammelt werden konnten.

Familie Parvocytheridae Hartmann, 1959

Gattung Parvocythere Hartmann, 1959

Parvocythere mauiensis sp. $\mathbf{n}$.

(Abb. 82-90)

Na m e: Nach dem Fundplatz der Art, der Insel Maui.

Hol otypus: Zergliederte Reste, ZIM K-34652

Locus typicus: Küstengrundwasserloch bei Honokowai, nördlich von Ka'anapali, $2 \mathrm{~m}$ vom Spülsaum, $40 \mathrm{~cm}$ tief in Grobsand.

Material: nur ein weibliches Exemplar.

B e s chreibung: Wegen der sehr charakteristischen Ausbildung von Schale und Extremitäten kann die Art beschrieben werden. Die Schalen zerrissen bei der Präparation, doch sind ihr Umriß und die Randverhältnisse gut erkennbar. Die Schalen sind im Umriß fast oval, besitzen am Vorderende jedoch eine den Schalenrand überragende Spitze in der LK. In der RK ist die Spitze, an der sich eine zweite Randzone ausbildet, nicht so deutlich. Die VZ ist schmal, Borsten stehen in relativ geringer Zahl am gesamten Schalenrand. Randkanäle waren nicht erkennbar. Die Au ist kräftig, die 5 letzten Glieder zylindrisch. Borsten wurden am IV (median-dorsal eine), am V (distal-ventral eine, dorsal-distal 2) und am Endglied (2 lange und eine kurze) festgestellt. Eine einzigartige Bildung ist eine Verbindungspange, die mit je einem Ast am Grundglied und am II ventral ansetzt und eine feste Verbindung zu Aa (Grenze von Grundglied und II dorsal) herstellt. Eine solche Verbindung ist dem Autor von keiner anderen Ostracoden-Art bekannt (vgl. Abb. 84/85). Aa kräftig, mit gedrungenen Gliedern. Am vorletzten Glied stehen median dorsal und ventral je eine Borste, ventral subdistal eine weitere kräftigere. Das fast quadratische Endglied trägt 2 Klauen. Die Spinnborste ist zweigeteilt, der distale kurze Teil ist deutlich schwächer als der Basisteil und läuft spitz zu. Taster der Md ebenfalls recht kräftig, II breit und kurz, III zylindrisch, Endglied distal verjüngt. Borsten befinden sich ventral-distal am Grundglied (eine), distal-dorsal und -ventral am II (je eine, median-ventral und -dorsal), am III (je eine), ventral-median am Endglied (eine) und 2 Endklauen. Die Atemplatte besitzt 2 Strahlen. Kaulade mit kurzen, spitzen Zähnen, die Lücken zwischen sich freilassen. Mxu mit schlanken Kfs und Taster. Borsten kurz und schwach, nur am Taster ist eine klauenartig verstärkt. Neben der Atemplatte steht ein dornenartiger Fortsatz. Beborstung der Beine abweichend von P. psammophila. Die Grundglieder der beiden Schreitbeine sind nach der Formel $121 / 011$ beborstet. Distal-dorsal konnte am II keine Borste nachgewiesen werden.

$\mathrm{Ma} B \mathrm{e}$ : Länge $0,217 \mathrm{~mm}$, Höhe $0,084 \mathrm{~mm}$.

Fundorte und Ökologie: s. Locus typicus.

Beziehungen: Von Hawaii ist bislang nur eine Parvocythere bekannt. Es ist dies P. psammophila. Diese ist deutlich kleiner und weicht auch im Bau der Extremitäten ab. Die neue Art gehört zwar zweifelsfrei zu den Parvocytheridae, steht aber durch 
Sonderbildungen (Verbindung Au/Aa, Beborstung der Schreitbeine) etwas isoliert. Auf die Arbeiten von Gottwald $(1980,1983)$ wird verwiesen.

\section{Cytheroidea incertae sedis}

Cytheride spec. 1 (Tafel 2: Fig. 16-17)

Cytheride spec. 2 (Tafel 2: Fig. 18-19)

In der aus einer Bachmündung bei Kekaha (Kauai) genommenen Probe fanden sich die Schalen von 2 Cytheriden-Arten, die der Autor nicht zuordnen kann. Sie werden hier aber abgebildet, um ihre Wiedererkennung zu ermöglichen.

Material: ZIM K-34653 (Scan-Teller).

Familie Paracyprididae Sars, 1923

Gattung Aglaiocypris Sylvester-Bradley, 1947

Aglaiocypris mauiensis sp. $\mathrm{n}$.

(Abb. 91-101)

N a m e: Nach dem Fundort der Art, der Insel Maui.

Hol o typus: zergliedert, ZIM K-34655 (ein Weibchen).

Paratypus: ZIM K-34655 (ein Weibchen). Steinen.

Locus typicus: Napili-Bay (NW-Seite der Insel Maui), Fluttümpel, Kies mit

Materia 1: zergliederter Holotypus und weiblicher, Paratypus.

B e s chreibung: Schalen gestreckt. VR und HR gerundet, VR gleichmäßiger als HR, der gegen den DR durch seine schwache Ecke abgesetzt ist. Weiteste Vorwölbung am HR und VR unter halber Höhe, beim HR etwas niedriger als beim VR. DR mit größter Höhe in der Mitte, schwach gekrümmt. VZ schmal, am VR stehen die Randkanäle dichter als am UR und HR. Au stark gekniet, mit weniger Gliedern (nur 4) als beim Generotypus (s. Diskussion der Beziehungen). I ventral mit 2 langen, auf einer gemeinsamen Basis entspringenden Borsten und einem stumpfen Anhang daneben. II ohne Bewehrung, III mit kräftigen Klauen ventral und einer Borste dorsal. Endglied mit 4 Borsten, die innere zweigeteilt. Aa mit großer Löffelborste proximal-ventral am II. Das vorletzte Glied und das kleine Endglied tragen distal 3 kräftige, lange Klauen und Borsten. Die Klauen sind länger als die beiden letzten Glieder zusammen. Md mit reich beborstetem Taster: I ventral 3, II distal-dorsal 2, -ventral 3, III distal-dorsal und -ventral je 3, Endglied mit 5 Borsten. An der Atemplatte stehen 3 Strahlen. Taster der Mxu mit 3 Borsten distal und 3 weiteren auf dem dorsalen Absatz. Die Atemplatte der Mxa springt deutlich vor und trägt 4 Strahlen. Endopodit mit blattartiger Spitze, vor der 2 feine Borsten stehen. Schreitbein ohne besondere Kennzeichen, mit langer Klaue (Abb. 98, 100). Putzfuß (Abb. 99) distal mit nach innen gerichteter glatter (?) Putzborste und einer weiteren, kurzen, distal gerichteten einfachen Borste. Fu mit 2 gezähnten Klauen und 2 Hinterrand-Borsten.

$\mathrm{M}$ a $B$ e : Länge $0,59-0,60 \mathrm{~mm}$, Höhe $0,26 \mathrm{~mm}$.

Fundorte und Ökologie: s. Locus typicus.

Beziehungen: Die Art steht der vom Autor (1974) beschriebenen aff. Aglaio- 
cypris eulitoralis sehr nahe. Auch bei dieser gibt es die gleichen Abweichungen vor allem an der Au. Keyser (1976) hat eine ebenfalls mit diesen Merkmalen versehene Art ais aff. Aglaiocypris eulitoralis floridiana aus Florida beschrieben. Die Florida-Unterart stammt aus Schill, die afrikanische eulitoralis wie die jetzt vorliegende mauiensis aus einem Rockpool. Es ist möglich, daß alle drei Arten/Unterarten in einer Spezies vereinigt werden können. Wir hätten es dann mit einer zirkumtropischen Art mit verschiedenen Unterarten zu tun. Die neue Art ist kleiner als eulitoralis. Die Zusammenlegung unterbleibt, bis mehr Material vorliegt. Weitere Aglaiocypris-Arten, die zu einem Vergleich herangezogen werden könnten, sind A. nipponica Okubo, 1980 aus Japan und $A$. croneisi Teeter, 1975. Auch diese beiden Arten stehen $A$. eulitoralis sehr nahe und könnten als Unterart derselben geführt werden. Okubos Art ist mit $0,7 \mathrm{~mm}$ aber deutlich größer als die neue Art. Teeter hat bedauerlicherweise keine Extremitäten abgebildet.

Familie Candodidae Kaufmann, 1900

Unterfamilie Thalassocypridinae Hartmann \& Puri, 1974

Gattung Dolerocypria Tressler, 1937

Dolerocypria minutissima sp. n.

(Abb. 102-110)

$\mathrm{N}$ a m e : minutissima, wegen der sehr geringen Größe der Art von nur 0,28 mm. Holotypu s: ZIM K-34654, ein Männchen.

L ocu s ty picu s: Hawaii (Big Island), Withington, Community Beach, Honu'apo Bay, SO-Seite der Insel; Tümpel in Lavastrom in Kontakt mit dem Meer, Steine mit Feinsand.

Material: s. Holotypus.

Beschreibung: Schale sehr flach und langgestreckt. HR schmal, VR etwas breiter gerundet. Die größte Höhe der Schale liegt etwas vor der Mitte. DR nur sehr flach gekrümmt. Auge deutlich. Hoden in der hinteren Schalenhälfte aufgerollt. VZ schmal, Randkanäle spärlich. Randverhältnisse nicht klar erkennbar (Schale entkalkt).

Au 6gliedrig, Grundglied kräftig. Der Endteil der Au besteht aus einer Serie schmaler, zylindrischer Glieder. Am II ist nur eine kurze Borste dorsal-distal, an den folgenden Gliedern dort jeweils 2 lange Sb. Endglied mit 3(?) Sb. Aa 5gliedrig. Grundglied und II umfangreich. Am Grundglied ventral eine Borste. Am II steht dorsal-proximal die Tastborste, ventral-proximal die Löffelborste und auf der distalen Kante 6 Borsten, davon 4 die Spitze der Endklauen überragende Schwimmborsten (Sb). Am III distal die terminal stumpf endenden beiden Männchenborsten, am IV distal-dorsal eine große Klaue, die das Ende der Endklauen des letzten Gliedes erreicht. Neben den Klauen des Endglieds eine einfache und eine messerförmige Borste. Md ohne wichtige Kennzeichen (s. Abb. 105), ebenso Mxu. Schreitbein mit sehr langer Klaue, innen bestachelt. Putzfuß terminal mit rückwärts gerichteter, zweigliedriger und distal einseitig gefiederter Putzborste, daneben 2 kleine distad gerichtete einfache Borsten. Fu mit 2 bestachelten Klauen, einer kurzen Vorderrand-Borste und 2 winzigen Hinterrand-Borsten. Kopulationsorgan birnförmig (Abb. 110). Im zentralen Spangenwerk ist eine Samenkapsel am inneren Rand sichtbar, die mit Spermatozoen angefüllt ist. Sie mündet in die Pumpe. Über dieser entspringt das sehr lange, mehrfach gewundene Begattungsrohr, dessen Ende schwer 
auszumachen ist, offenbar im äußeren Spangenwerk sich in Richtungsrohren verliert (?). Ductus ej. mit 6 Ringen von Chitinstiften (Abb. 109).

$\mathrm{Ma}$ B e : Länge $0,28 \mathrm{~mm}$, Höhe $0,084 \mathrm{~mm}$.

Fundorte und Ökologie: s. Locus typicus.

Beziehungen: Die Art ist die wohl kleinste der Gattung. Sehr ähnlich im Umriß und Bau der Extremitäten ist Dolerocypria bifurcata Maddocks, 1986, doch ist das Kopulationsorgan des Männchens bei dieser Art abweichend gebaut. D. inopinata Klie, 1939 unterscheidet sich durch Fu und Kopulationsorgan, D. mukaishinensis Okubo, 1980 ist viel größer und hat einen abweichenden Umriß. D. taalensis weicht ebenfalls im Schalenbau ab.

Familie Pontocyprididae G. W. Müller, 1894

Pontocypridide spec. Ma 11/2

Ein leider sehr stark mazeriertes Männchen einer offenbar neuen Art kam in einer Probe vom Black-Sand-Strand südlich Lahaina (Westseite von Maui) vor. Gleichfalls eine Larve dieser Familie fand sich am Strand der Kiholo Bay (nördlich Kailina, Kona).

Danksagung. Der Autor dankt Herrn H. Kunz für die Überlassung des Materials, Frau E. Quintero für die Anfertigung der Tintenzeichnungen, Frau H. Mielke und Frau S. Zeckert für die Erstellung der Reinschrift des Manuskripts und Frau R. Walter für die Anfertigung der REM-Aufnahmen und REM-Tafeln.

\section{LITERATUR}

Brady, G. S., 1880. Report on the Ostracoda dredged by H.M.S. Challenger during the years 1873-1876. - Zoology 1, 1-184.

Brady, G. S., 1890. On Ostracoda collected by H. B. Brady, Esq., L.L.D., F.R.S., in the South Sea Islands. - Trans. R. Soc. Edinb. 35, 489-525.

Gottwald, J., 1980. Systematik, postembryonale Entwicklung, Evolution und Zoogeographie interstitieller Ostracoda (Crustacea) aus dem Pazifik. Diss., Univ. Göttingen, $260 \mathrm{pp}$.

Gottwald, J., 1983. Interstitielle Fauna von Galàpagos XXX. Podocopida I (Ostracoda). - Mikrofauna Meeresboden 90, 1-187.

Hartmann, G., 1974. Zur Kenntnis des Eulitorals der afrikanischen Westküste zwischen Angola und Kap der Guten Hoffnung und der afrikanischen Ostküste von Südafrika und Moçambique unter besonderer Berücksichtigung der Polychaeten und Ostracoden. Teil 3. Die Ostracoden des Untersuchungsgebiets. - Mitt. hamb. zool. Mus. Inst. 69 (Erg. Bd), 229-520.

Hartmann, G., 1984. Zur Kenntnis der Ostracoden der polynesischen Inseln Huahiné (Gesellschaftsinseln) und Rangiroa (Tuamotu-Inseln). - Mitt. hamb. zool. Mus. Inst. 81, 117-169.

Holden, J. C., 1967: Late cenozoic ostracodes from the drowned terraces in the Hawaiian Islands. Pacif. Sci. 21 (1), 1-50.

Holden, J. C., 1976. Late cenozoic Ostracoda from Midway Island drill holes. - Prof. Pap. geol. Surv. $680-F, 1-43$.

Keyser, D., 1976. Zur Kenntnis der brackigen Weichböden Südwest-Floridas unter besonderer Berücksichtigung ihrer Ostracodenfauna. Diss., Univ. Hamburg, 191 pp.

Kornicker, L. S., 1976. Benthic marine Cypridinacea from Hawaii (Ostracoda). - Smithson. Contr. Zool. 231, 1-24. 


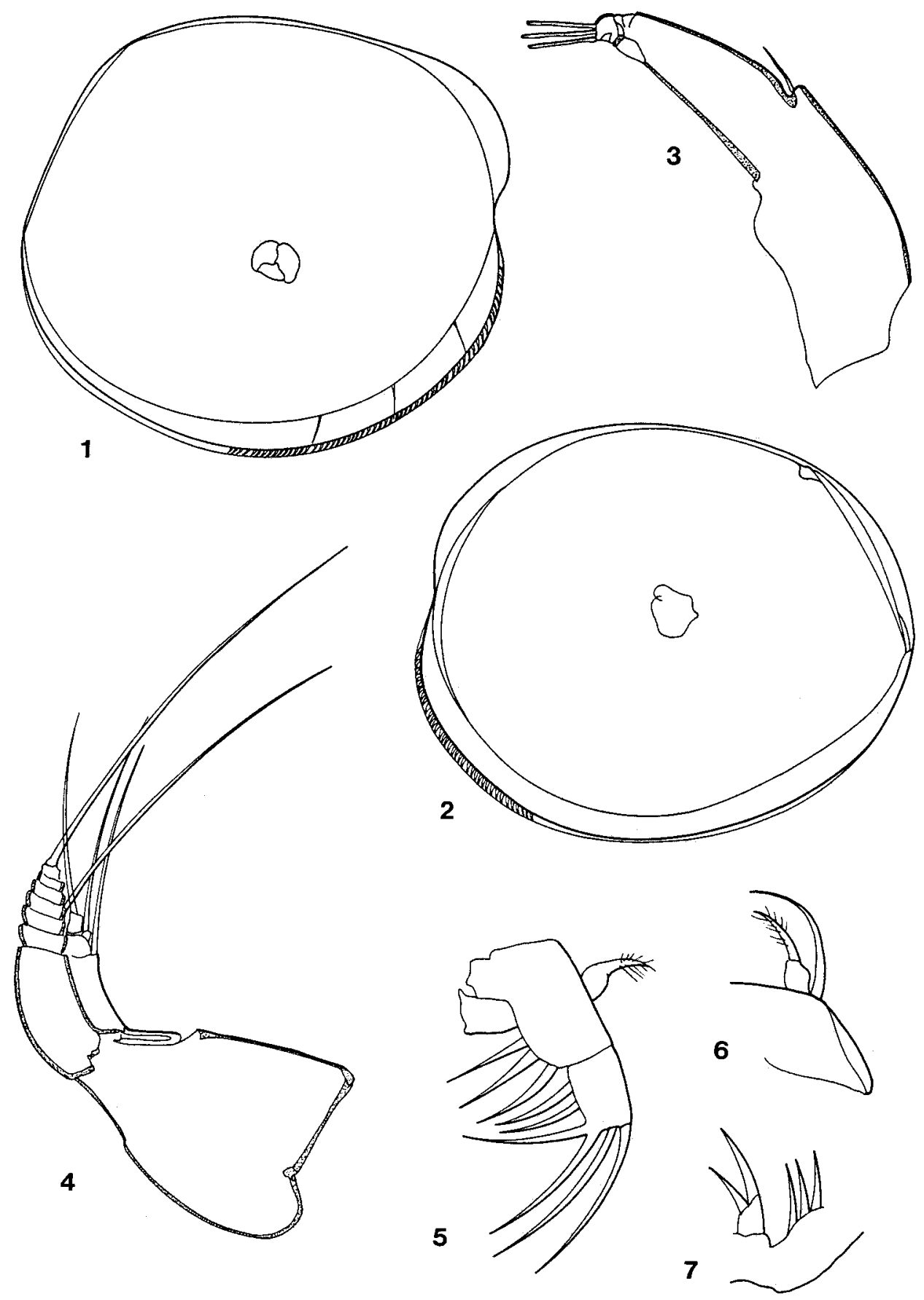

Abb. 1-7. Polycope hawaiiensis sp. n. 1: RK. 2: LK. 3: Au. 4: Aa. 5: Md, Taster. 6: Md, Exp. 7: Md, Kaulade 

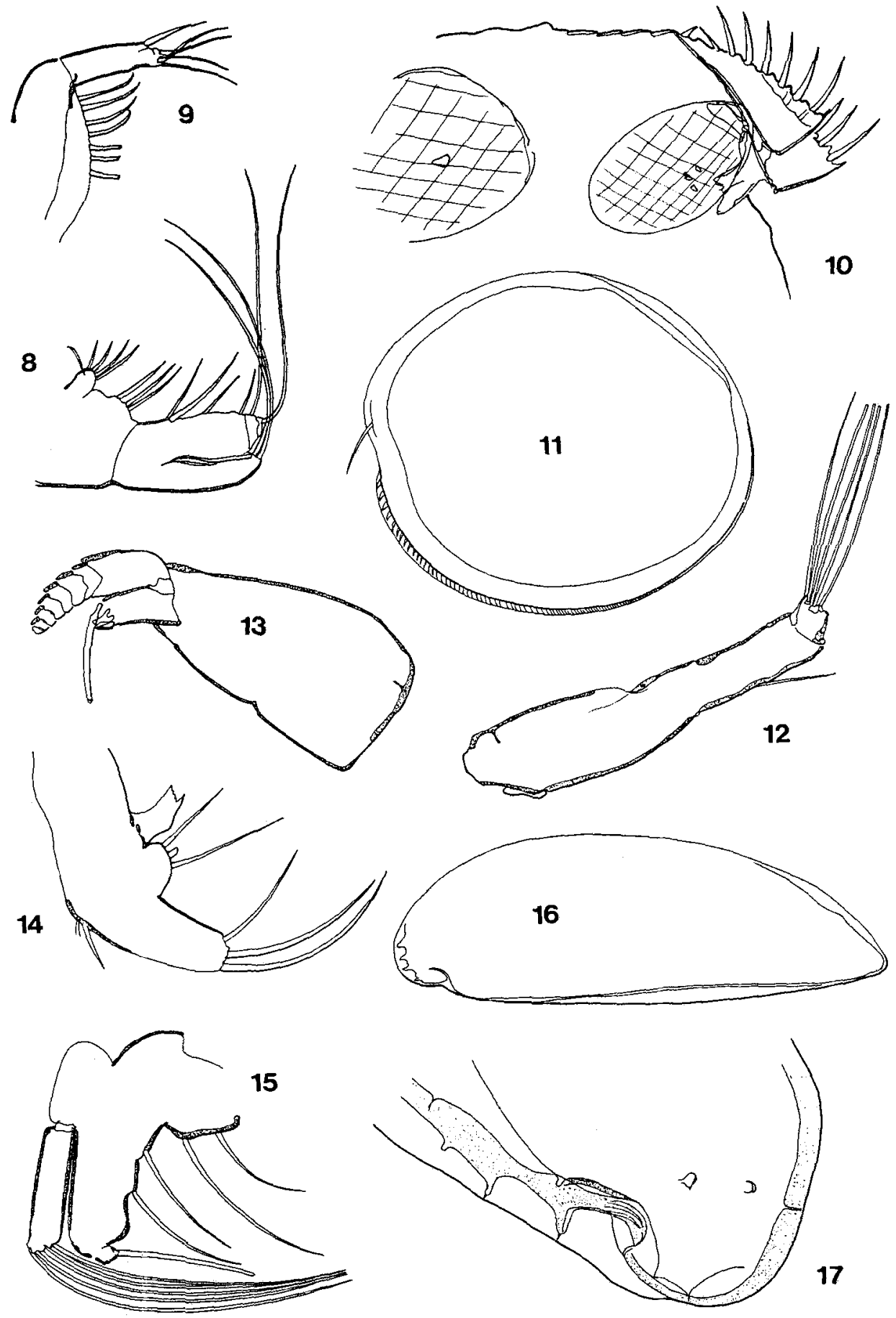

Abb. 8-10. Polycope hawaiiensis sp. n. 8: Mxu. 9: Mxa. 10: Furca

Abb. 11-15. Polycope (? Polycopiella) spec. Maui 11. 11: LK. 12: Au. 13: Aa. 14: Md. 15: Mxu Abb. 16-17. Cobanocythere konensis sp. n. 16: LK. 17: VR 


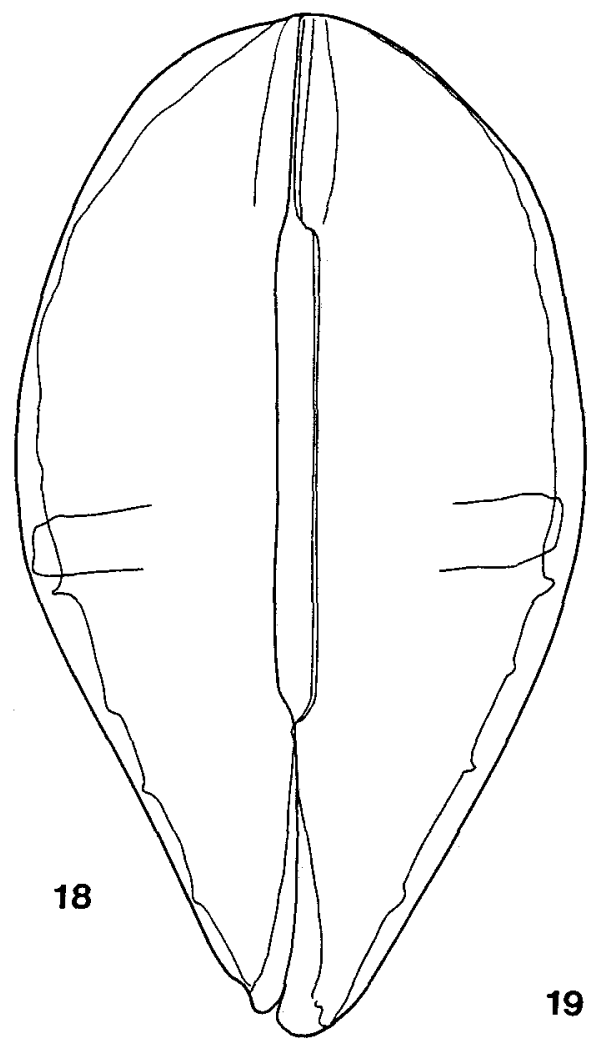

19
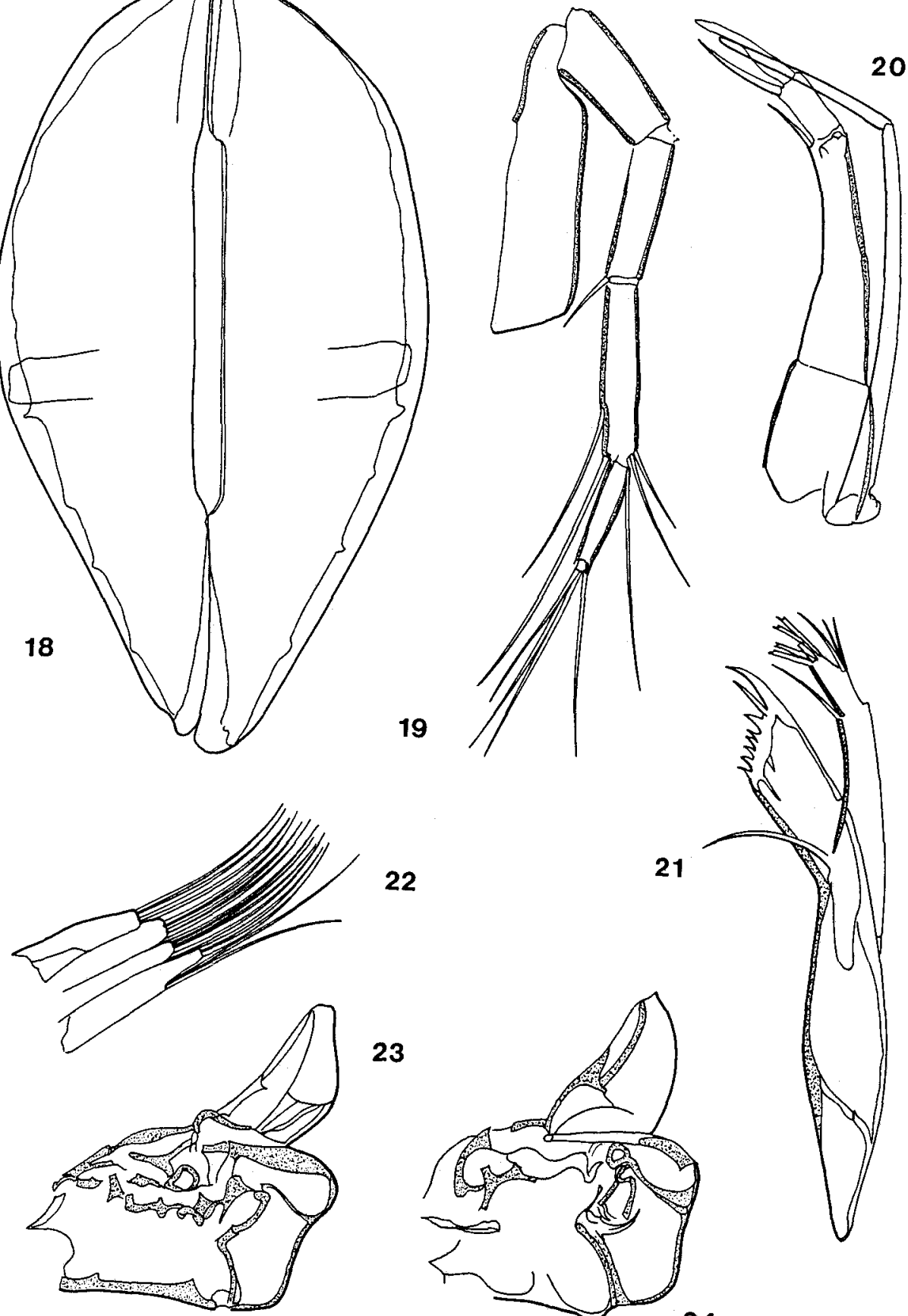

22

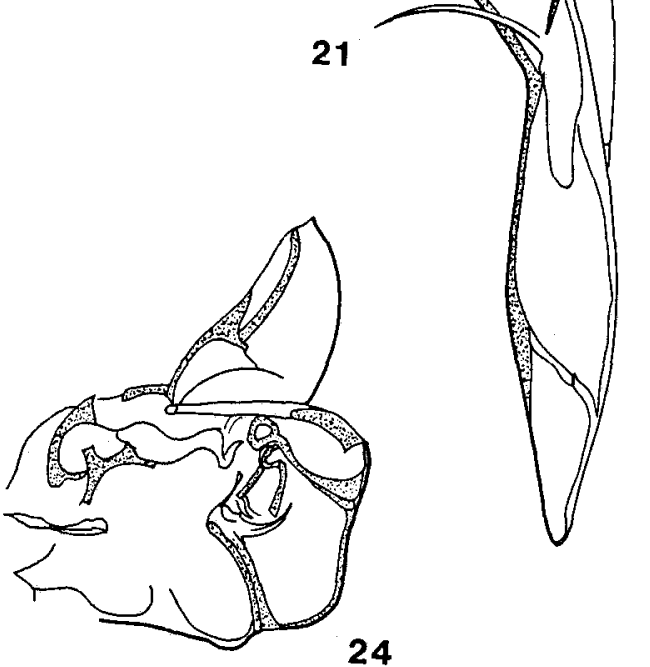

Abb. 18-24. Cobanocythere konensis sp. n. 18: Muschel von oben. 19: Au. 20: Aa. 21: Md. 22: Mxu. 23, 24: Kop.-Organ des Männchens 

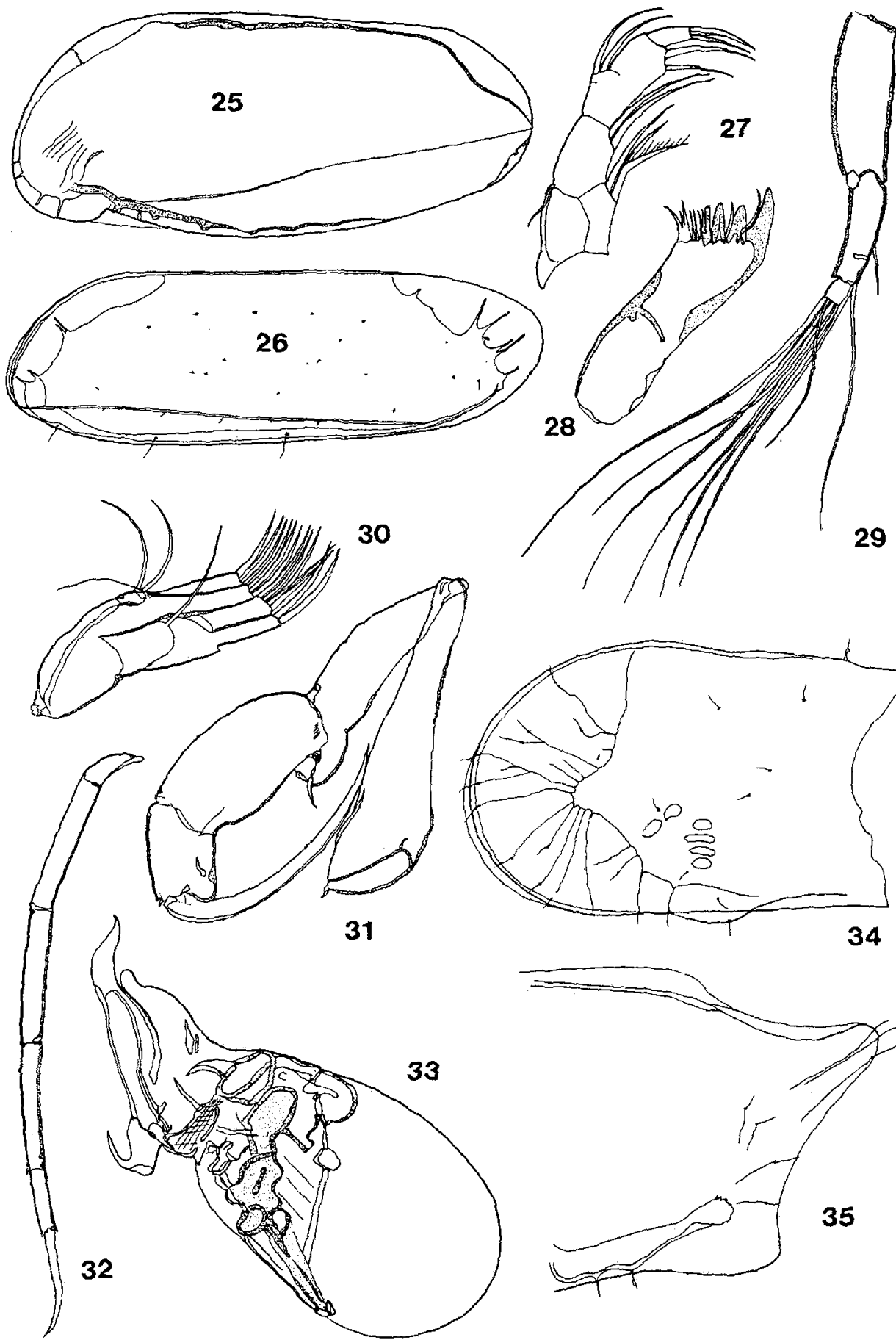

Abb. 25. Cobanocythere spec. HW 18: LK

Abb: 26-33. Psammocythere hawaiiensis sp. n. 26: L.K. 27: Md, Taster. 28: Md, Kaulade. 29:

Au. 30: Mxu. 31: Mxa, Greifer. 32: P : 33: Kop.-Organ des Männchens

Abb. 34-35. Cytheropteron spec. Maui 16. 34: LK, Vorderende. 35: LK, Hinterende 

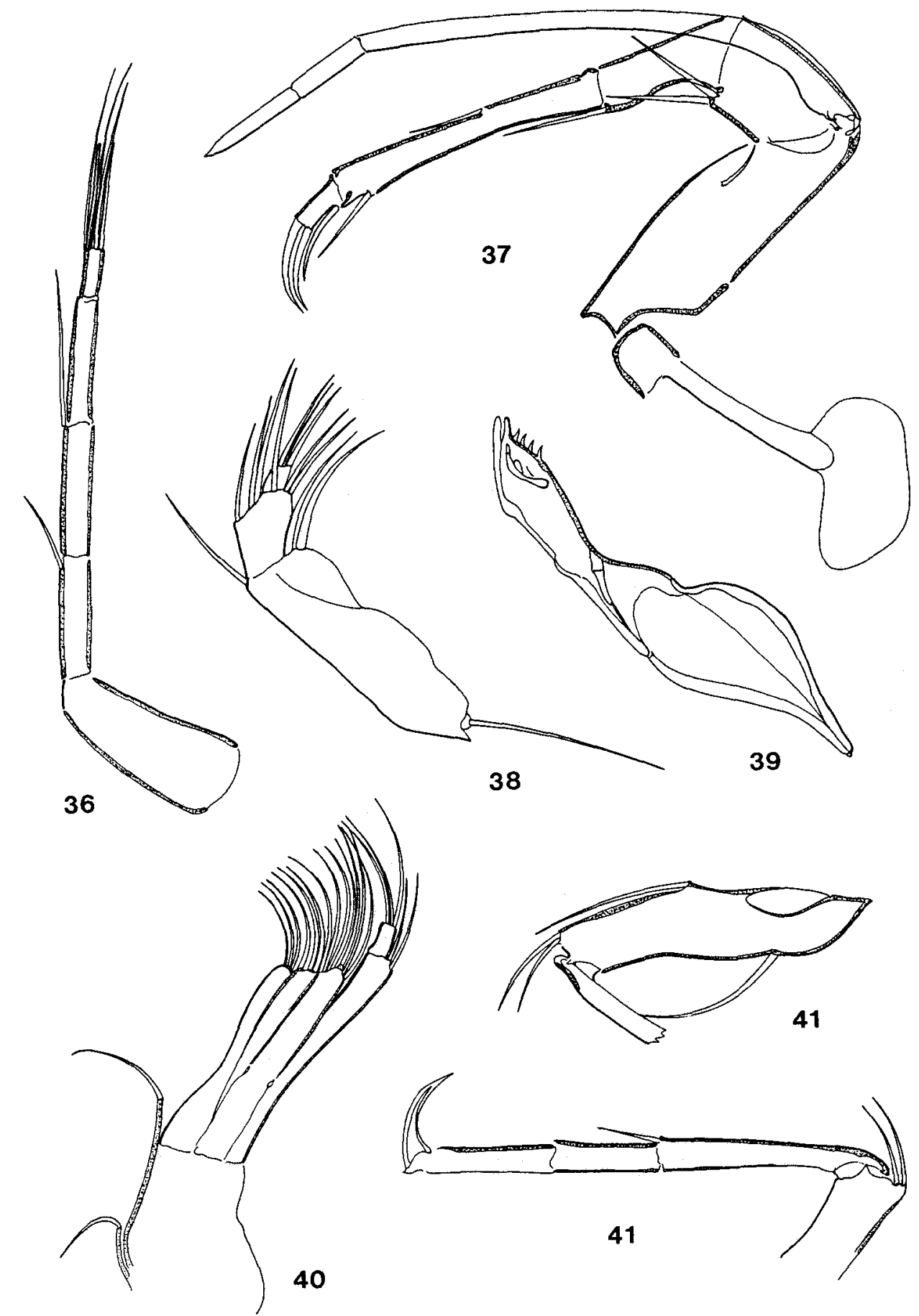

Abb. 36-41. Cytheropteron spec. Maui 16. 36: Au. 37: Aa. 38: Md, Taster. 39: Md, Stamm und Kaulade. 40: Mxu. 41: P 1, Basis und Schreitteil 

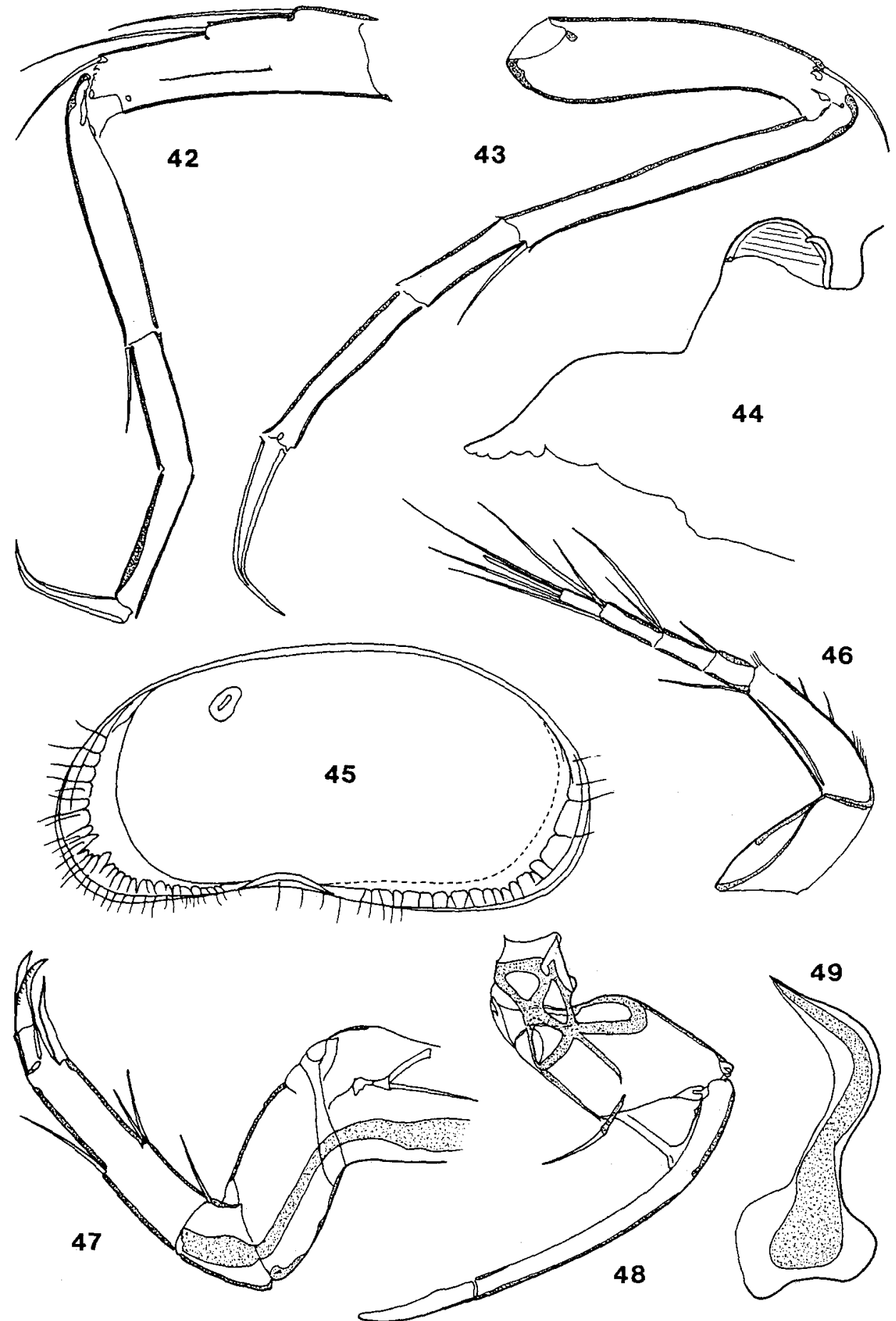

Abb. 42-44. Cytheropteron spec. Maui 16. 42: P 2. 43: P 3. 44: Hinterende des Weibchens Abb. 45-49. Xestoleberis hawaiiensis sp. n. 45: LK. 46: Au. 47: Aa, Stamm. 48: Aa, Spinnborste. 49: Aa, Spinndrüse 

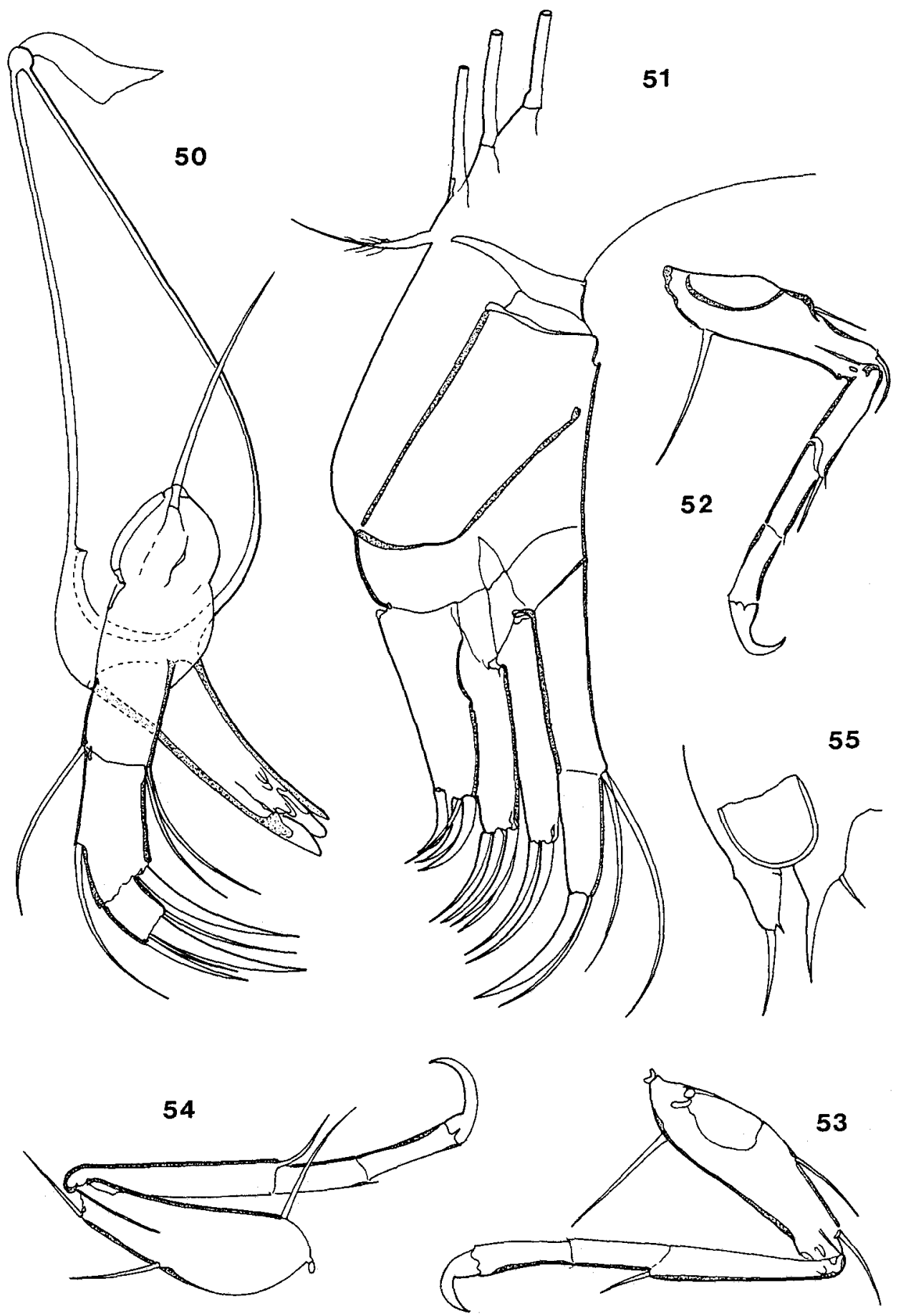

Abb. 50-55. Xestoleberis hawaiiensis sp. n. 50: Md. 51: Mxu. 52: P 1. 53: P 2. 54: P 3. 55: Körperende des Weibchens 

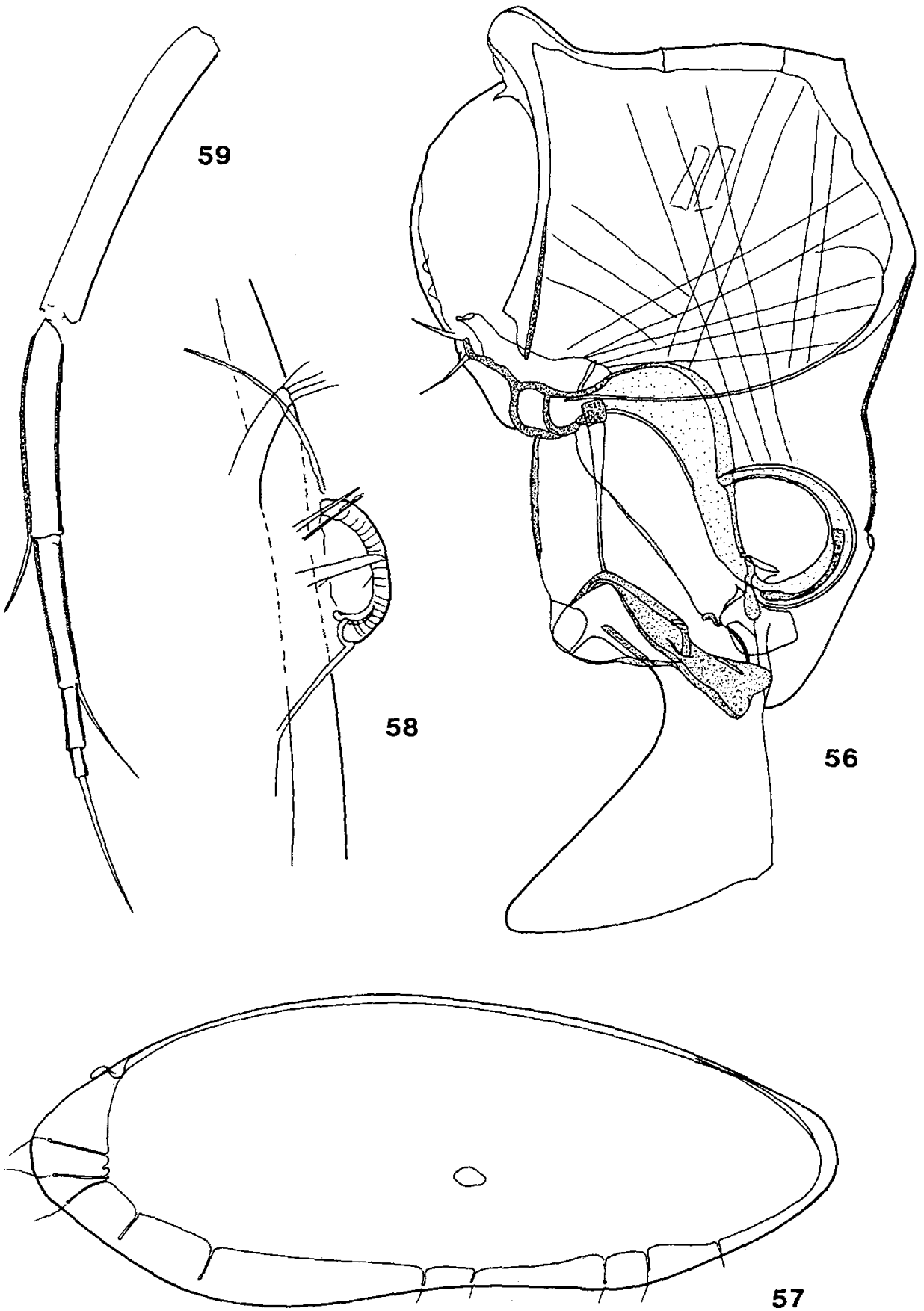

Abb. 56. Xestoleberis hawailensis sp. n., Kop.-Organ des Männchens Abb. 57-59. Paradoxostoma kunzi sp. n. 57: RK. 58: Mundscheibe. 59: Au 

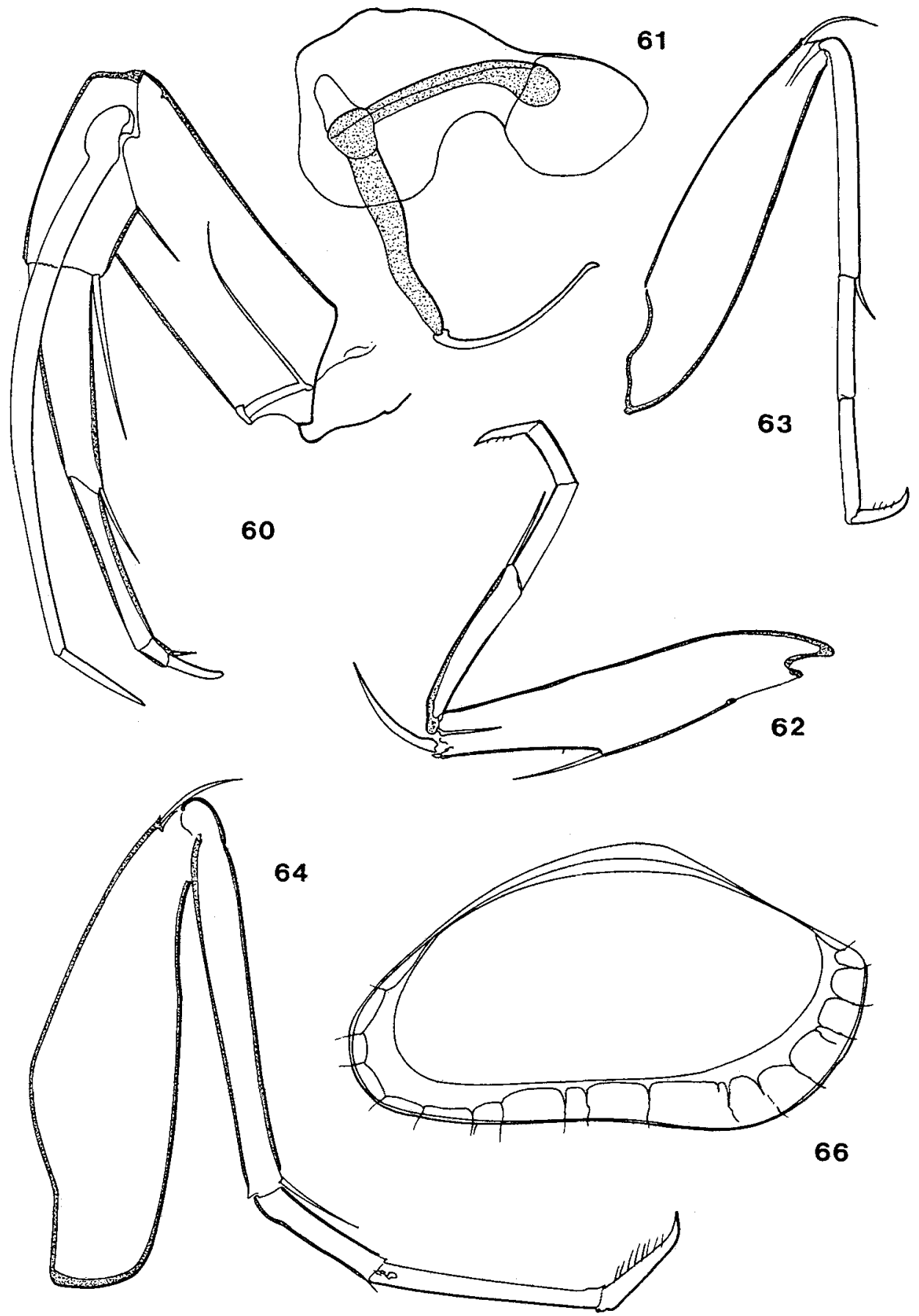

Abb. 60-64. Paradoxostoma kunzi sp. n. 60: Aa. 61: Aa, Spinndrüse. 62: P 1. 63: P 2. 64: P 3 Abb. 66. Paradoxostoma kauaiensis sp. n., LK des Weibchens 

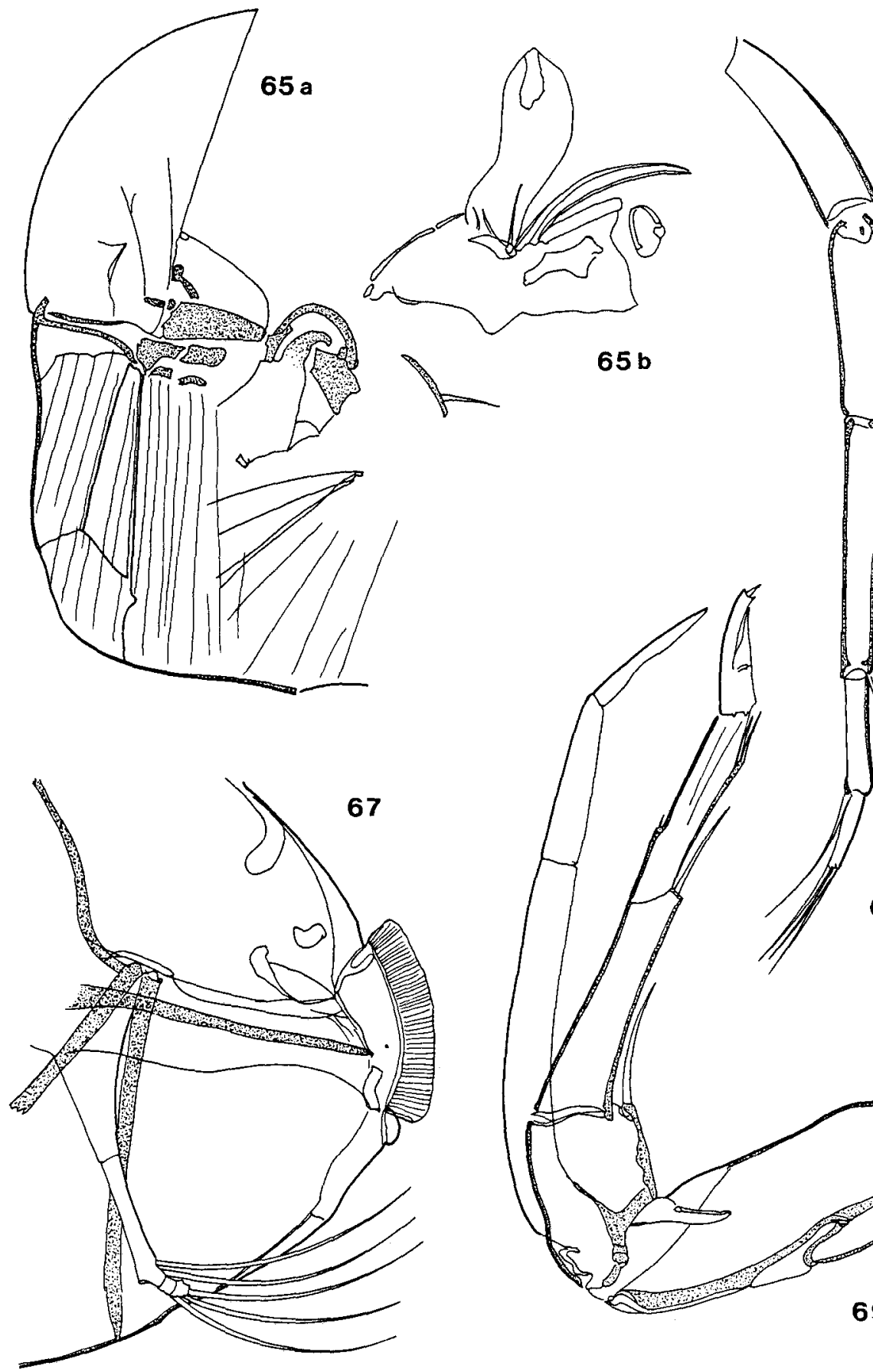


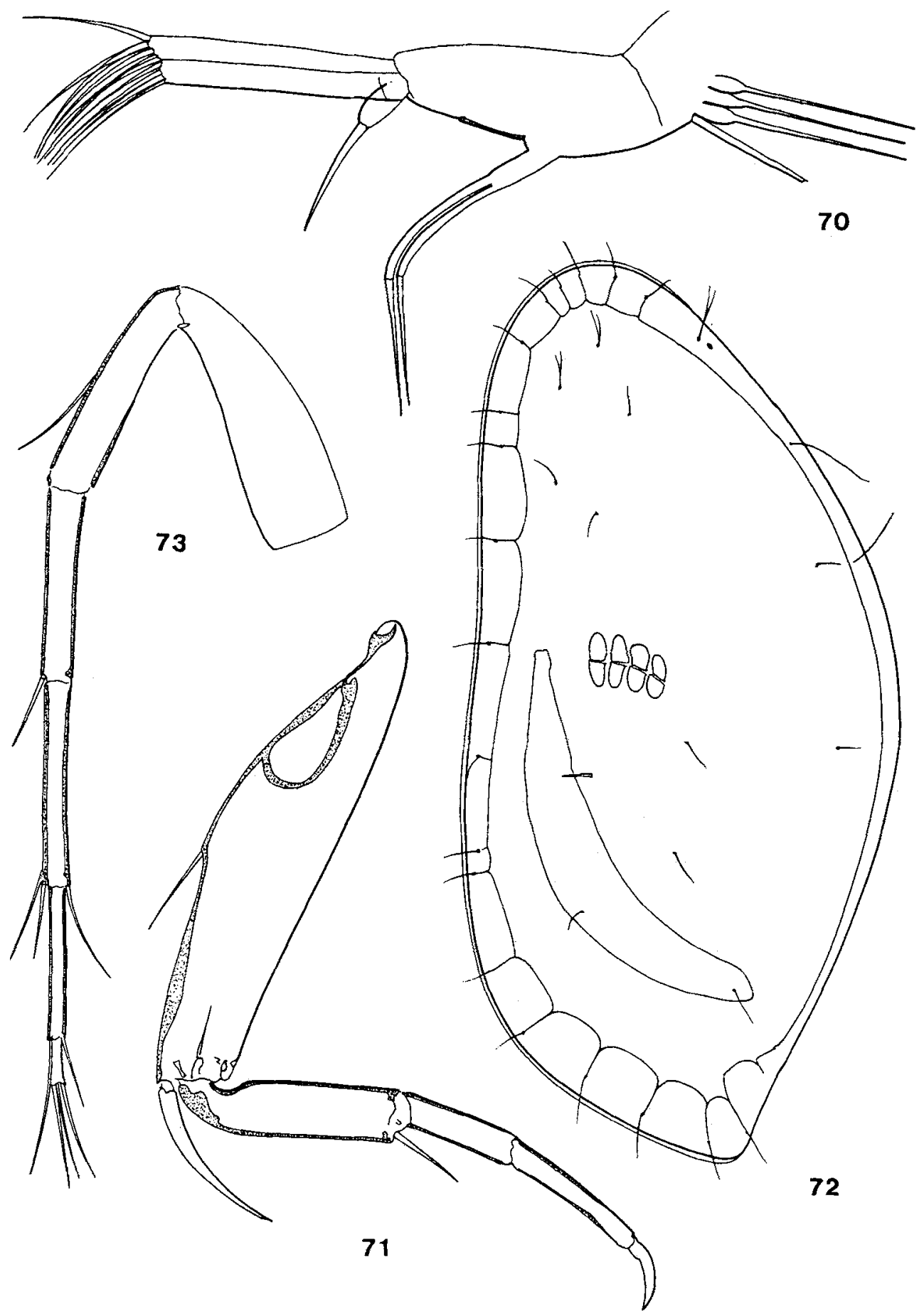

Abb. 70-73. Paradoxostoma kauaiensis sp. n. 70: Mxu des Weibchens. 71: P 1 des Weibchens. 72: LK des Männchens. 73: Au des Männchens 


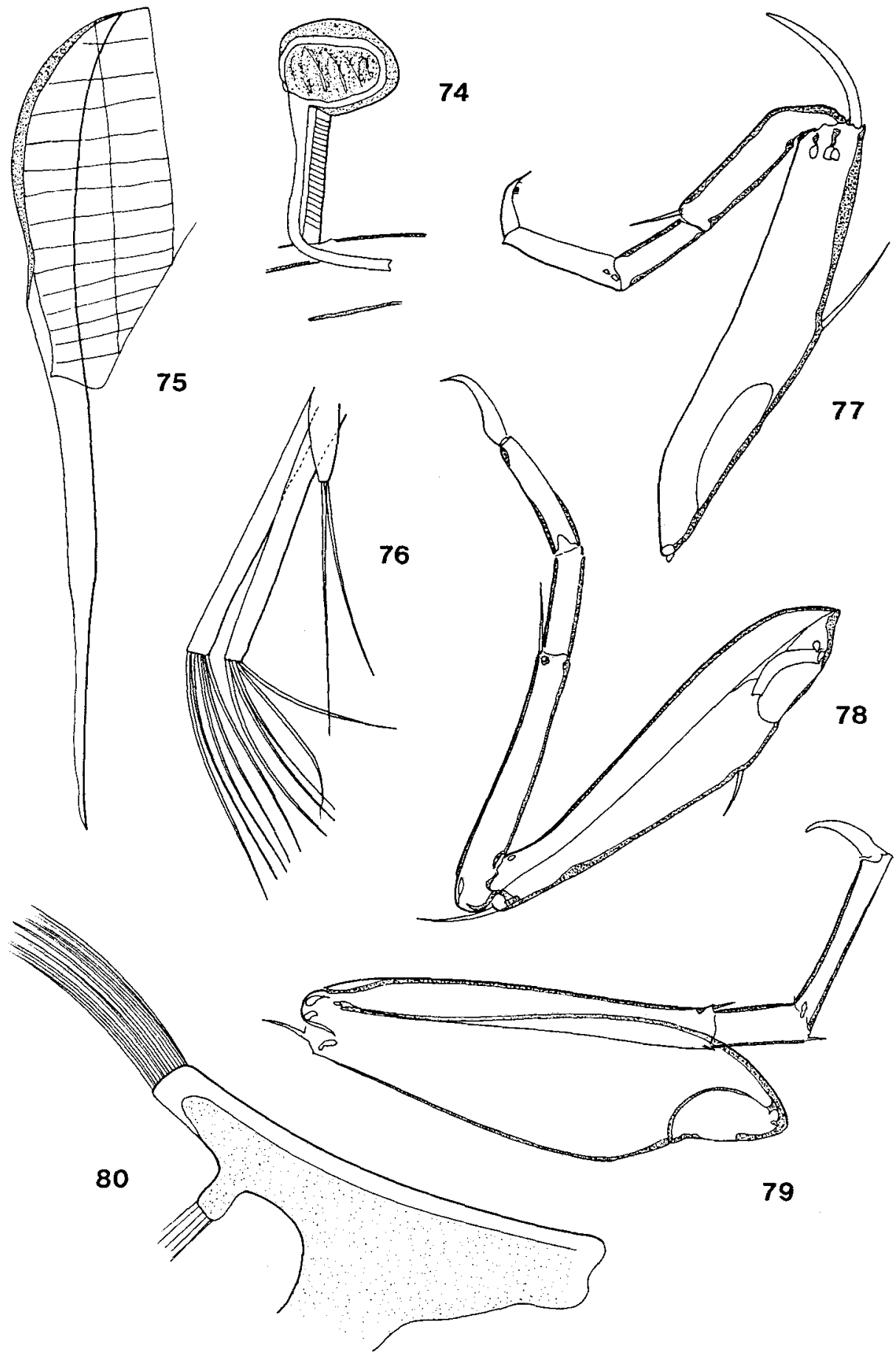

Abb. 74-80. Paradoxostoma kauaiensis sp. n. 74: Aa, Spinndrüse. 75: Md, Stechborste. 76: Mxu des Männchens. 77: P 1 des Männchens. 78: P 2 des Männchens. 79: P 3 des Männchens. 80: Bürstenförmiges Organ des Männchens 

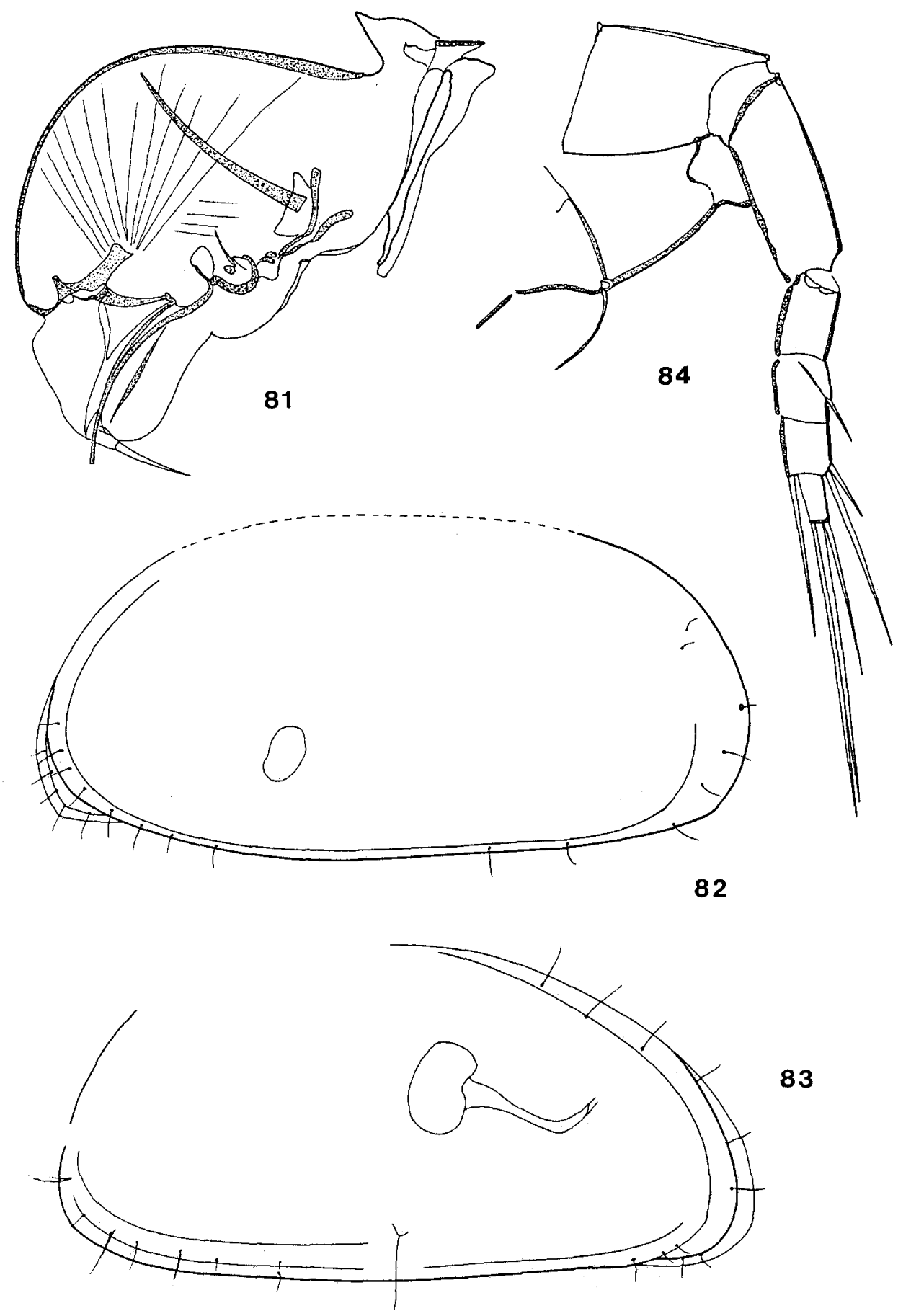

Abb. 81. Paradoxostoma kauaiensis sp. n., Kop.-Organ des Männchens Abb. 82-84. Parvocythere mauiensis sp. n. 82: LK. 83: RK. 84: Au 

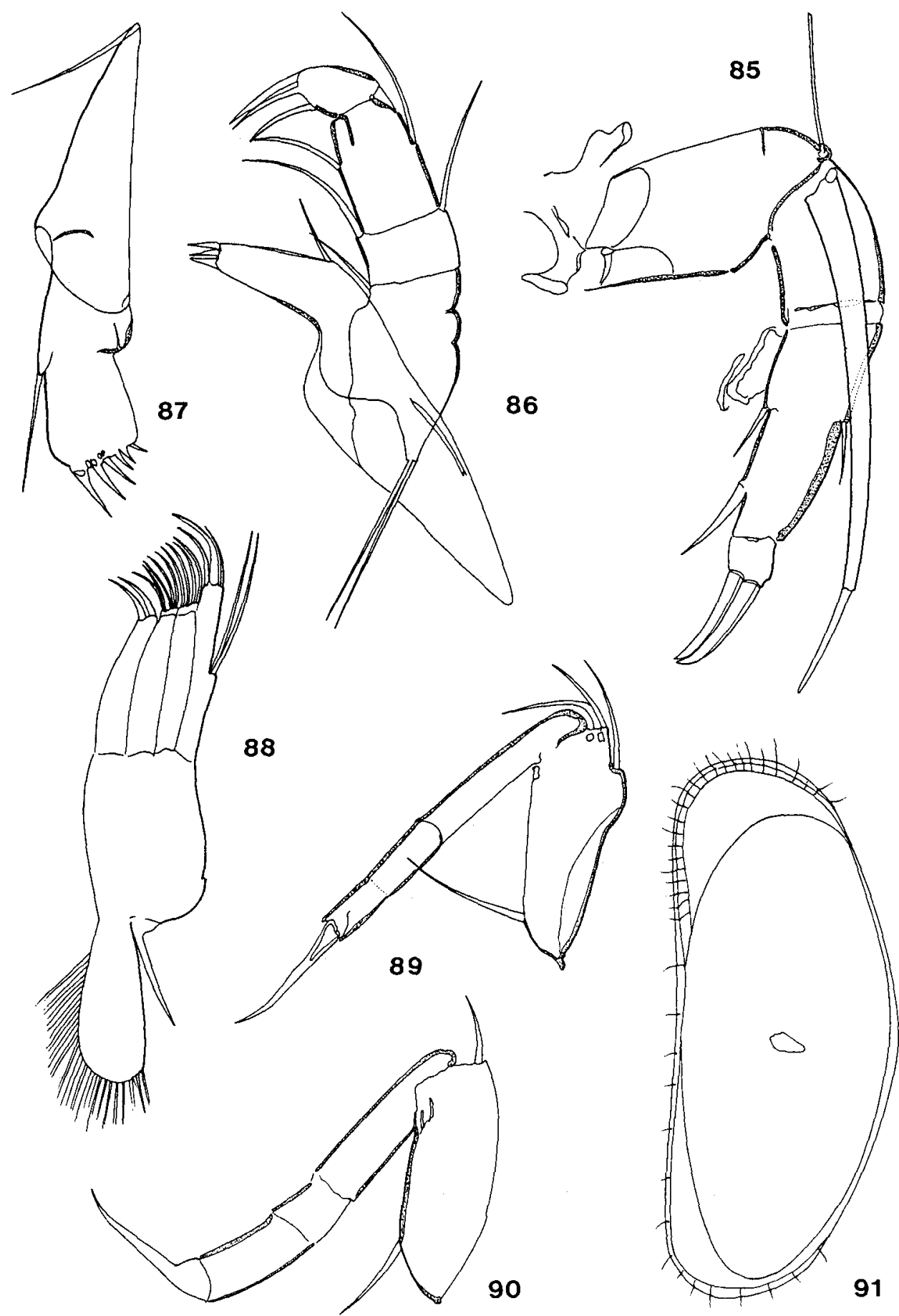


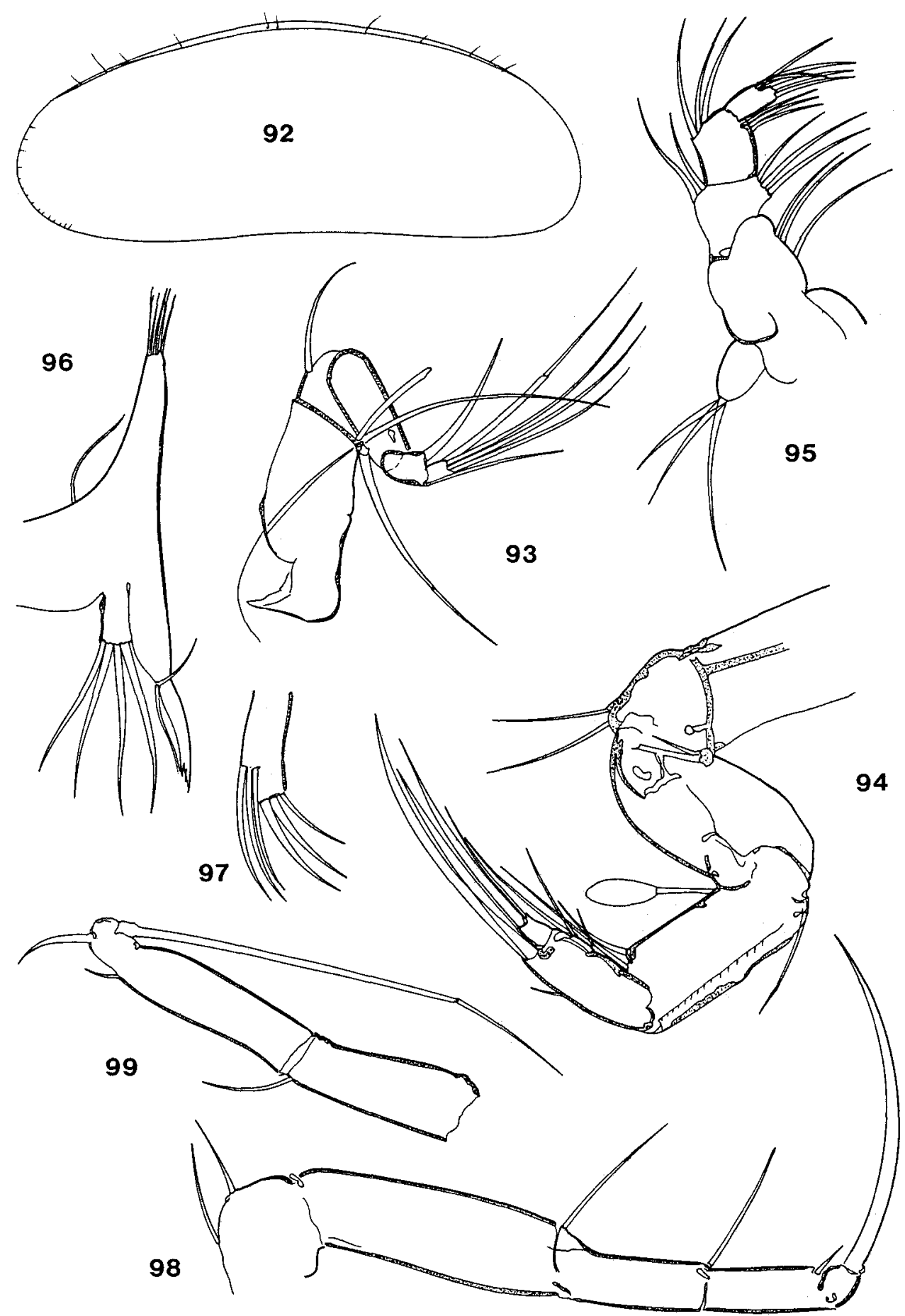

Abb. 92-98. Aglaiocypris mauiensis sp. n. 92: RK, Umriß. 93: Au. 94: Aa. 95: Md. 96: Mxa. 97: Mxu, Taster. 98: P 1. 99: P 2, Putzfuß 

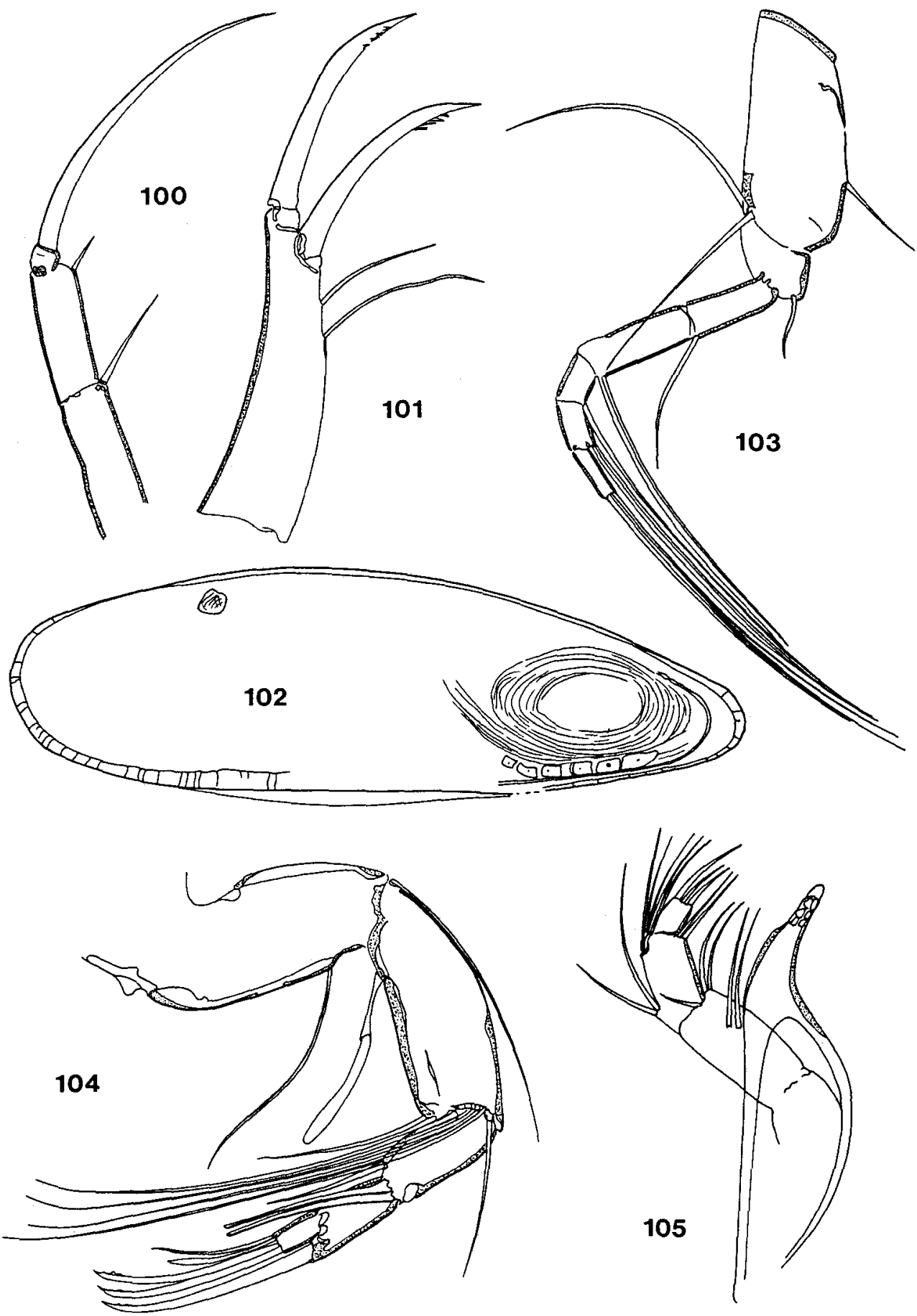

Abb. 100-101. Aglaiocypris mauiensis sp. n. 100: P 1, Endborste. 101: Furca Abb. 102-105. Dolerocypria minutissima sp. n. 102: LK. 103: Au. 104: Aa. 105: Md 


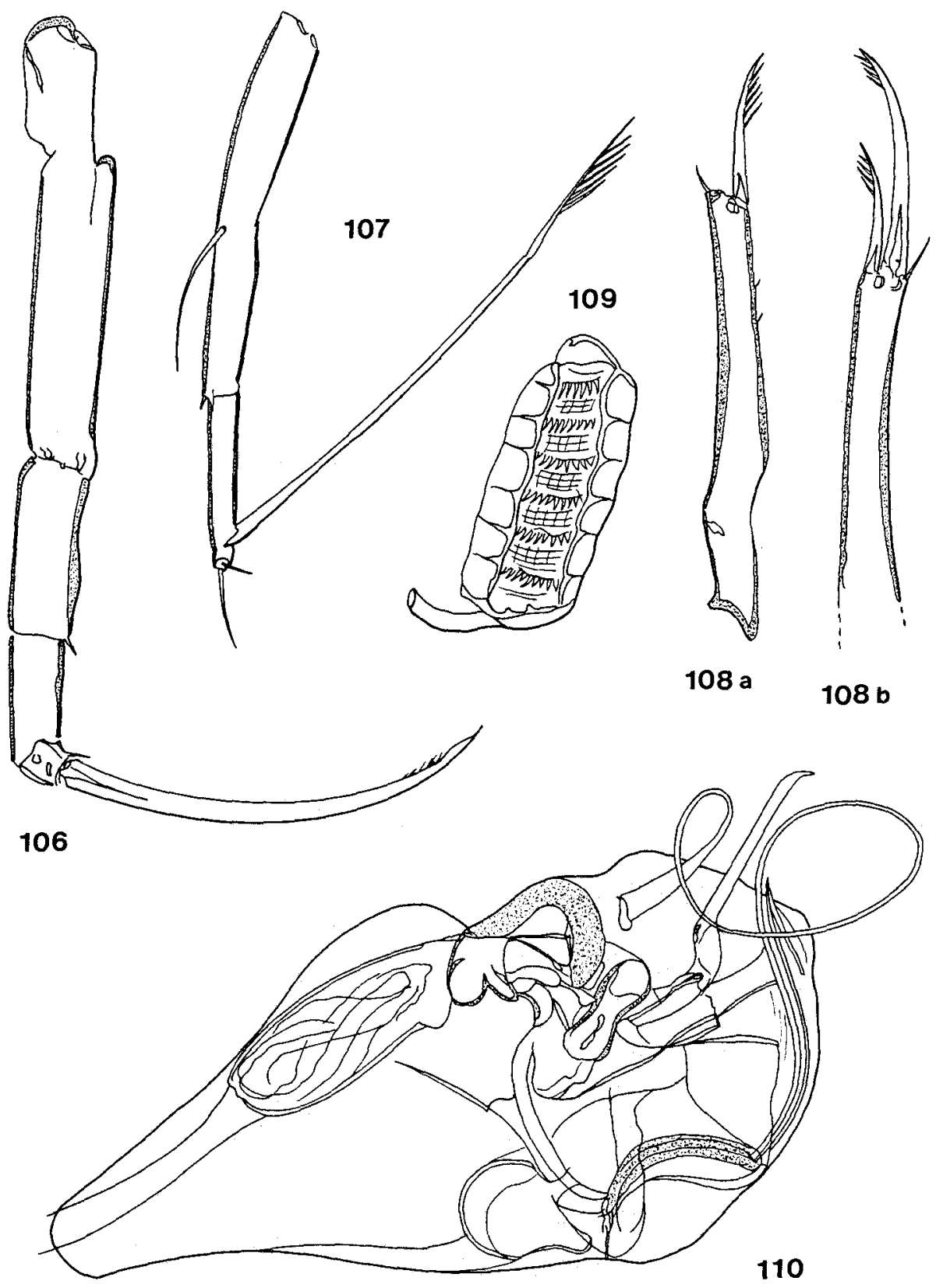

Abb. 106-110. Dolerocypria minutissima sp. n. 106: P 1. 107: P 2 (Putzfuß). 108a, b: Furcaäste. 109: Ductus ejaculatorius. 110: Kop.-Organ des Männchens 


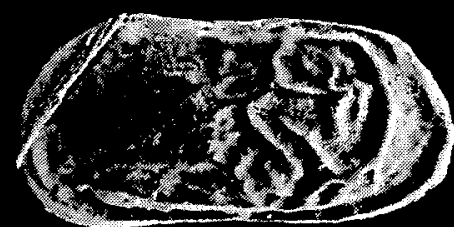

1

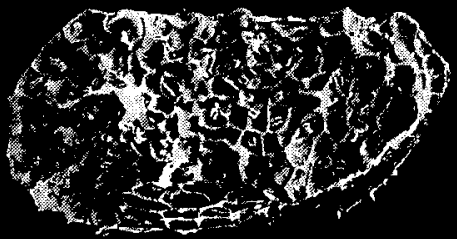

3

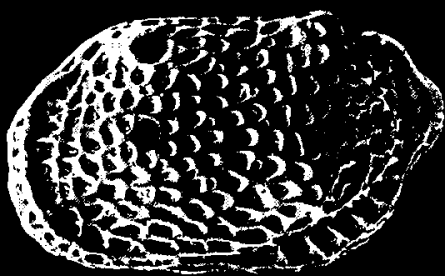

5

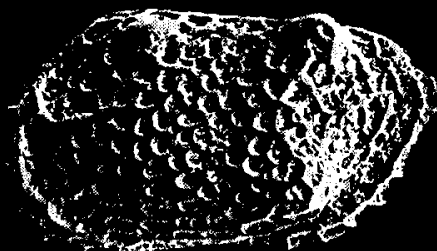

7

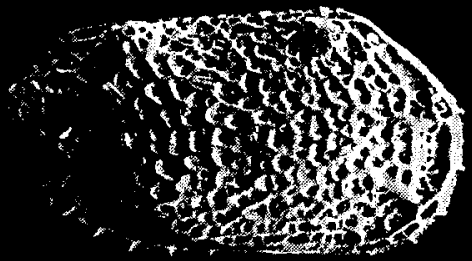

9

$30 \mathrm{man}$

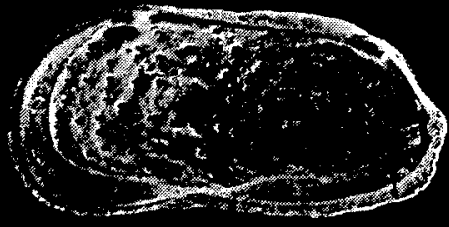

2
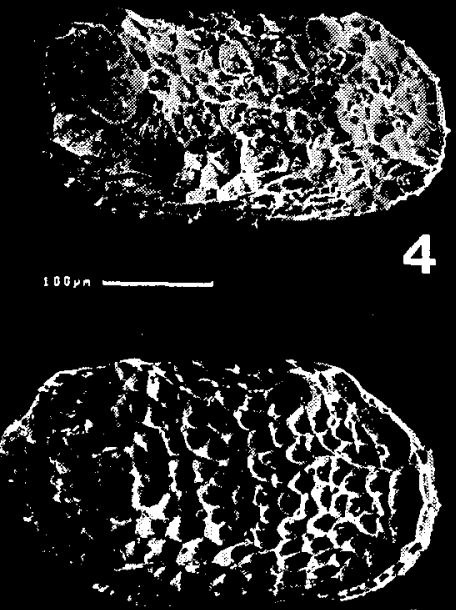

6

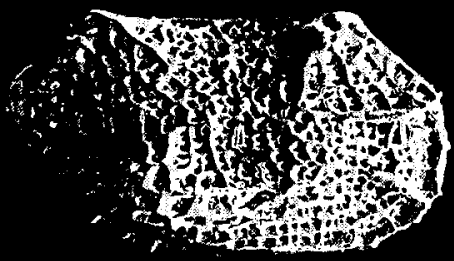

$103 \times n$

8

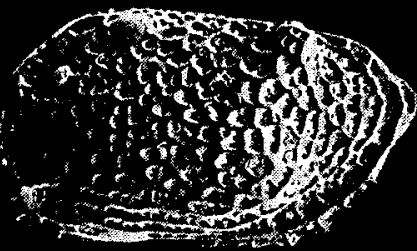

10

Tafel 1. Fig. 1-2. Hemicytheride spec. Ka 29. 1: LK von außen. 2: RK von innen Fig. 3-4. Loxoconcha spec. Ka 3. 3: LK von außen. 4: RK von außen

Fig. 5-10. Loxoconcha spec. Ka 1. 5: LK von außen, adult. 6: RK von außen, adult. 7 u. 10: LK von außen, larval. 8 u. 9: RK von außen, larval 


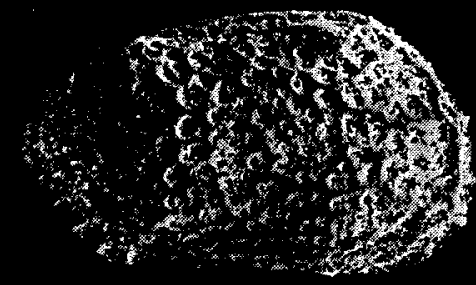

11

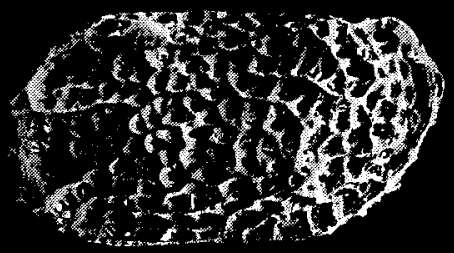

13

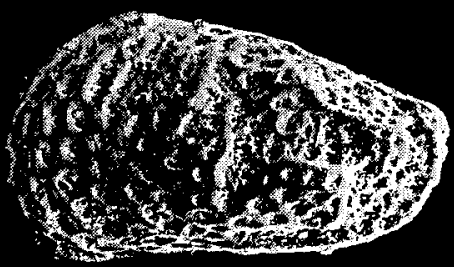

$14 b$

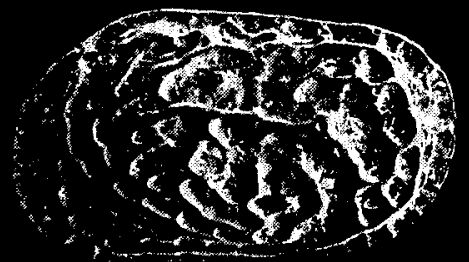

16

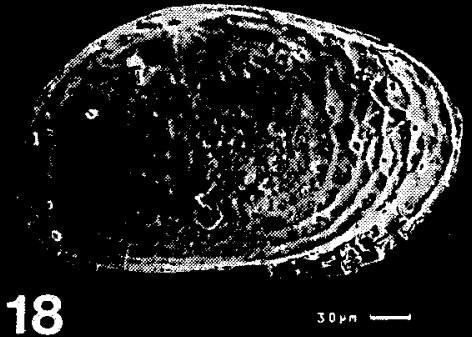

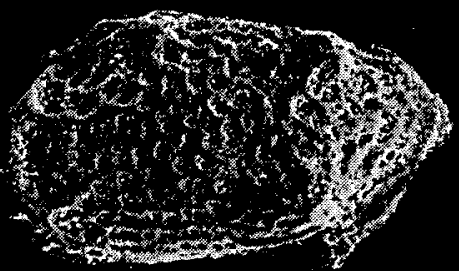

12

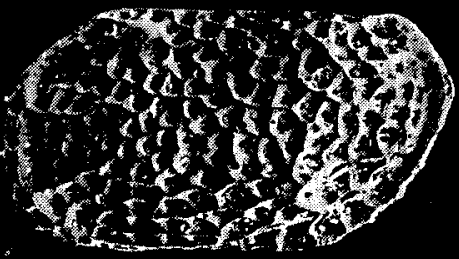

$14 a$
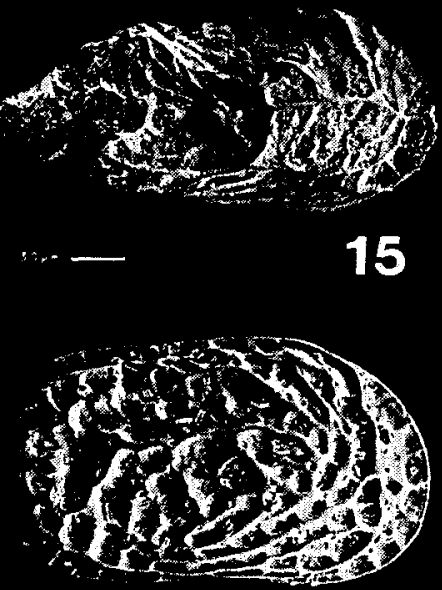

17

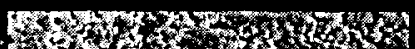
1.

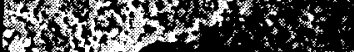
1.

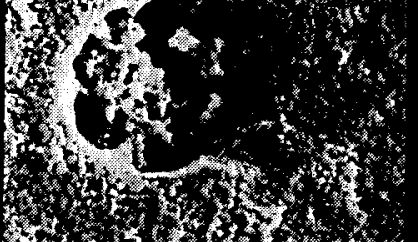
(3)

19

Tafel 2. Fig. 11-12. Loxoconcha spec. Ka 1. 11: RK von außen, larval. 12: LK von außen, larval Fig. 13 u. 14 a. Loxoconcha spec. Ka 2, LKK von außen

Fig. 14b. Morkhovenia inconspicua (Brady, 1880), LK von außen

Fig. 15. Paracytheridea spec. Kaui 29, RK von außen

Fig. 16-17. Cytheride spec. 1. 16: LK von außen. 17: RK von außen

Fig. 18-19. Cytheride spec. 2. 18: LK von außen. 19: Porenkanal 